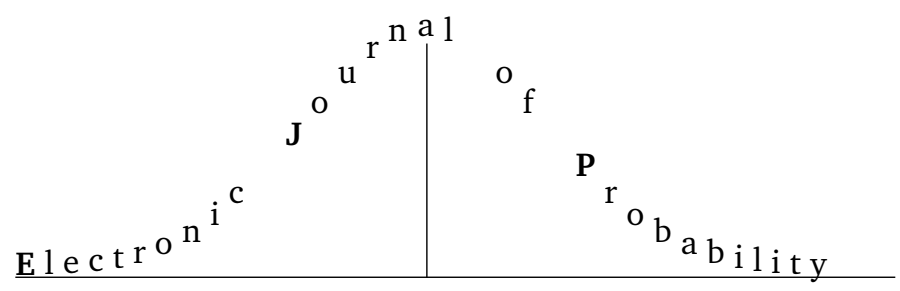

Vol. 13 (2008), Paper no. 64, pages 1952-1979.

Journal URL

http://www .math.washington.edu/ ejpecp/

\title{
Positively and negatively excited random walks on integers, with branching processes
}

\author{
Elena Kosygina* \\ Department of Mathematics \\ Baruch College - CUNY \\ One Bernard Baruch Way \\ New York, NY 10010, USA \\ elena.kosygina@baruch.cuny.edu
}

\author{
Martin P.W. Zerner \\ Mathematisches Institut \\ Universität Tübingen \\ Auf der Morgenstelle 10 \\ 72076 Tübingen, Germany \\ martin.zerner@uni-tuebingen.de
}

\begin{abstract}
We consider excited random walks on $\mathbb{Z}$ with a bounded number of i.i.d. cookies per site which may induce drifts both to the left and to the right. We extend the criteria for recurrence and transience by M. Zerner and for positivity of speed by A.-L. Basdevant and A. Singh to this case and also prove an annealed central limit theorem. The proofs are based on results from the literature concerning branching processes with migration and make use of a certain renewal structure.
\end{abstract}

Key words: Central limit theorem, excited random walk, law of large numbers, positive and negative cookies, recurrence, renewal structure, transience.

AMS 2000 Subject Classification: Primary 60K35, 60K37, 60J80.

Submitted to EJP on January 13, 2008, final version accepted October 14, 2008.

\footnotetext{
*E. Kosygina's work was partially supported by the CUNY Research Foundation, PSC-CUNY award \# 69580-00-38.
} 


\section{Introduction}

We consider nearest-neighbor random walks on the one-dimensional integer lattice in an i.i.d. cookie environment with a uniformly bounded number of cookies per site. The uniform bound on the number of cookies per site will be denoted by $M \geq 1, M \in \mathbb{N}$. Informally speaking, a cookie environment is constructed by placing a pile of cookies at each site of the lattice (see Figure 1). The piles of cookies represent the transition probabilities of the random walker: upon each visit to a site the walker consumes the topmost cookie from the pile at that site and makes a unit step to the right or to the left with probabilities prescribed by that cookie. If the cookie pile at the current site is empty the walker makes a unit step to the right or to the left with equal probabilities.

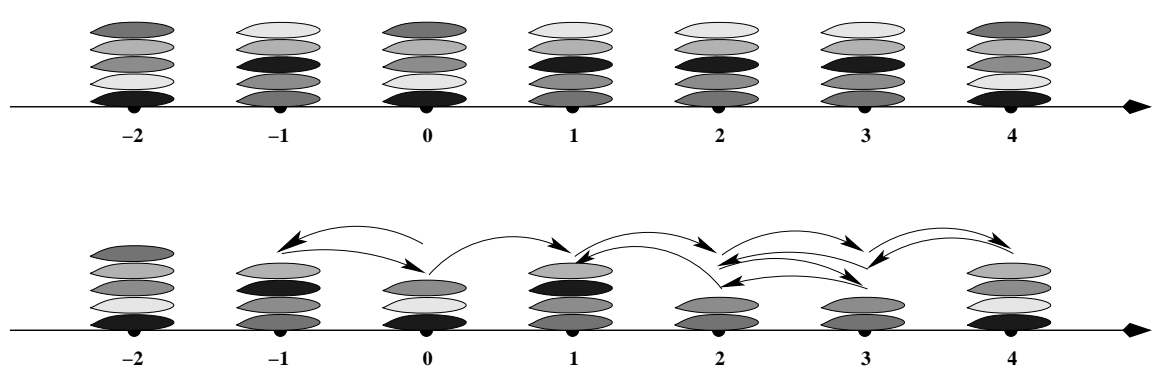

Figure 1: The top picture is an example of an i.i.d. cookie environment with $M=5$, which consists of two types of cookie piles. An independent toss of a fair coin determines which type of cookie pile is placed at each site of the lattice. Various shades of gray allude to different transition probabilities associated to different cookies. The bottom picture shows the first few possible steps of a random walker in this cookie environment starting at 0.

A cookie will be called positive (resp. negative) if its consumption makes the walker to go to the right with probability larger (resp. smaller) than $1 / 2$. A cookie which is neither positive nor negative will be called a placebo. Placebo cookies allow us to assume without loss of generality that each pile originally consists of exactly $M$ cookies. Unless stated otherwise, the random walker always starts at the origin.

The term "excited random walk" was introduced by Benjamini and Wilson in [BW03], where they considered random walks on $\mathbb{Z}^{d}, d \geq 1$, in an environment of identical cookies, one per each site. Allowing more (or fewer) than one cookie per site and randomizing the environment naturally gave rise to the multi-excited random walk model in random cookie environments. We refer to [Zer05] and [Zer06] for the precise description and first results. It was clear then that this new model exhibits a very interesting behavior for $d=1$. We shall mention some of the results for $d \geq 2$ in Section 9 and now concentrate on the one-dimensional case.

The studies of excited random walks on integers were continued in [MPV06], [BS08a], and [BS08b]. [AR05] deals with numerical simulations of this model. In all papers mentioned above a (possible) bias introduced by the consumption of a cookie was assumed to be only in one direction, say, positive. The recurrence and transience, strong law of large numbers [Zer05], conditions for positive linear speed [MPV06], [BS08a], and the rates of escape to infinity for transient walks with zero speed [BS08b] are now well understood. Yet some of the methods and facts used in the proofs (for example, comparison with simple symmetric random walks, submartingale property) depend significantly on this "positive bias" assumption.

The main novelty of the current paper is in considering cookie environments, which may induce positive or negative drifts at different sites or even at the same site on successive visits. Our main results 
are the recurrence/transience criterion (Theorem 1), the criterion for positive linear speed (Theorem 2) and an annealed central limit theorem (Theorem 3). The first two theorems are extensions of those for non-negative cookie environments but we believe that this is a purely one-dimensional phenomenon. Moreover, in Section 9 we give an example, which shows that, at least for $d \geq 4$, the criteria for recurrence or transience and for positive linear speed can not depend just on a single parameter, the average total drift per site (see (3)). The order of the cookies in the pile should matter as well.

The proofs are based on the connections to branching processes with migration. Branching processes allowing both immigration and emigration were studied by several authors in the late 70-ties through about the middle of the 90-ties, and we use some of the results from the literature (Section 2). See the review paper [VZ93] for more results and an extensive list of references up to about 1990. The connection between one-dimensional random walks and branching processes was observed long time ago. In particular, it was used for the study of random walks in random environments, see e.g. [KKS75]. In the context of excited random walks, this idea was employed recently in [BS08a], [BS08b] (still under the "positive bias" assumption). In the present paper we are using results from the literature about branching processes with migration in a more essential way than [BS08a] and [BS08b]. One of our tasks is to show how to translate statements about excited random walks into statements for a class of branching processes with migration which have been studied in the past.

Let us now describe our model, which we shall abbreviate by ERW, more precisely. A cookie environment $\omega$ with $M$ cookies per site $z \in \mathbb{Z}$ is an element of

$$
\begin{aligned}
& \Omega_{M}:=\left\{\left((\omega(z, i))_{i \in \mathbb{N}}\right)_{z \in \mathbb{Z}} \mid \omega(z, i) \in[0,1], \forall i \in\{1,2, \ldots, M\}\right. \\
&\text { and } \omega(z, i)=1 / 2, \forall i>M, \forall z \in \mathbb{Z}\} .
\end{aligned}
$$

The purpose of $\omega(z, i)$ is to serve as the transition probability from $z$ to $z+1$ of a nearest-neighbor ERW upon the $i$-th visit to a site $z$. More precisely, for fixed $\omega \in \Omega_{M}$ and $x \in \mathbb{Z}$ an ERW $\left(X_{n}\right)_{n \geq 0}$ starting from $x$ in the cookie environment $\omega$ is a process on a suitable probability space with probability measure $P_{x, \omega}$ which satisfies:

$$
\begin{aligned}
P_{x, \omega}\left[X_{0}=x\right] & =1, \\
P_{x, \omega}\left[X_{n+1}=X_{n}+1 \mid\left(X_{i}\right)_{0 \leq i \leq n}\right] & =\omega\left(X_{n}, \#\left\{i \leq n \mid X_{i}=X_{n}\right\}\right), \\
P_{x, \omega}\left[X_{n+1}=X_{n}-1 \mid\left(X_{i}\right)_{0 \leq i \leq n}\right] & =1-\omega\left(X_{n}, \#\left\{i \leq n \mid X_{i}=X_{n}\right\}\right) .
\end{aligned}
$$

The cookie environment $\omega$ may be chosen at random itself according to a probability measure on $\Omega_{M}$, which we shall denote by $\mathbb{P}$, with the corresponding expectation operator $\mathbb{E}$. Unless stated otherwise, we shall make the following assumption on $\mathbb{P}$ :

$$
\text { The sequence }(\omega(z, \cdot))_{z \in \mathbb{Z}} \text { is i.i.d. under } \mathbb{P} \text {. }
$$

Note that assumption (1) does not imply independence between different cookies at the same site but only between cookies at different sites, see also Figure 1. To avoid degenerate cases we shall also make the following mild ellipticity assumption on $\mathbb{P}$ :

$$
\mathbb{E}\left[\prod_{i=1}^{M} \omega(0, i)\right]>0 \text { and } \mathbb{E}\left[\prod_{i=1}^{M}(1-\omega(0, i))\right]>0 .
$$


After consumption of a cookie $\omega(z, i)$ the random walk is displaced on $P_{x, \omega}$-average by $2 \omega(z, i)-1$. This average displacement, or drift, is positive for positive cookies and negative for negative ones. The consumption of a placebo cookie results in a symmetric random walk step. Averaging the drift over the environment and summing up over all cookies at one site defines the parameter

$$
\delta:=\mathbb{E}\left[\sum_{i \geq 1}(2 \omega(0, i)-1)\right]=\mathbb{E}\left[\sum_{i=1}^{M}(2 \omega(0, i)-1)\right],
$$

which we shall call the average total drift per site. It plays a key role in the classification of the asymptotic behavior of the walk as shown by the three main theorems of this paper.

Our first result extends [Zer05, Theorem 12] about recurrence and transience for non-negative cookies to i.i.d. environments with a bounded number of positive and negative cookies per site.

Theorem 1 (Recurrence and transience). If $\delta \in[-1,1]$ then the walk is recurrent, i.e. for $\mathbb{P}$-a.a. environments $\omega$ it returns $P_{0, \omega}$-a.s. infinitely many times to its starting point. If $\delta>1$ then the walk is transient to the right, i.e. for $\mathbb{P}$-a.a. environments $\omega, X_{n} \rightarrow \infty$ as $n \rightarrow \infty P_{0, \omega}$-a.s.. Similarly, if $\delta<-1$ then the walk is transient to the left, i.e. $X_{n} \rightarrow-\infty$ as $n \rightarrow \infty$.

Trivial examples with $M=1$ and $\omega(0,1)=0$ or $\omega(0,1)=1$ show that assumption (2) is essential for Theorem 1 to hold.

Our next result extends [MPV06, Theorem 1.1, Theorem 1.3] and [BS08b, Theorem 1.1] about the positivity of speed from spatially uniform deterministic environments of non-negative cookies to i.i.d. environments with positive and negative cookies.

Theorem 2 (Law of large numbers and ballisticity). There is a deterministic $v \in[-1,1]$ such that the excited random walk satisfies for $\mathbb{P}$-a.a. environments $\omega$,

$$
\lim _{n \rightarrow \infty} \frac{X_{n}}{n}=v \quad P_{0, \omega} \text {-a.s.. }
$$

Moreover, $v<0$ for $\delta<-2, v=0$ for $\delta \in[-2,2]$ and $v>0$ for $\delta>2$.

While Theorems 1 and 2 give necessary and sufficient conditions for recurrence, transience, and the positivity of the speed, the following central limit theorem gives only a sufficient condition. To state it we need to introduce the annealed, or averaged, measure $P_{x}[\cdot]:=\mathbb{E}\left[P_{x, \omega}[\cdot]\right]$.

Theorem 3 (Annealed central limit theorem). Assume that $|\delta|>4$. Let $v$ be the velocity given by Theorem 2 and define

$$
B_{t}^{n}:=\frac{1}{\sqrt{n}}\left(X_{\lfloor t n\rfloor}-\lfloor t n\rfloor v\right) \quad \text { for } t \geq 0 .
$$

Then $\left(B_{t}^{n}\right)_{t \geq 0}$ converges in law under $P_{0}$ to a non-degenerate Brownian motion with respect to the Skorohod topology on the space of cadlag functions.

The variance of the Brownian motion in Theorem 3 will be further characterized in Section 6 , see (28).

Let us describe how the present article is organized. Section 2 introduces the main tool for the proofs, branching processes with migration, and quotes the relevant results from the literature. In 
Sections 3 and 4 we describe the relationship between ERW and branching processes with migration and introduce the necessary notation. In Section 5 we use this relationship to translate results from Section 2 about branching processes into results for ERW concerning recurrence and transience, thus proving Theorem 1. In Section 6 we introduce a renewal structure for ERW, similar to the one which appears in the study of random walks in random environments (RWRE), and relate it to branching processes with migration. In Sections 7 and 8 we use this renewal structure to deduce Theorems 2 and 3, respectively, from results stated in Section 2. The final section contains some concluding remarks and open questions.

Throughout the paper we shall denote various constants by $c_{i} \in(0, \infty), i \geq 1$.

\section{Branching processes with migration - results from the literature}

In this section we define a class of branching processes with migration and quote several results from the literature. We chose to give the precise statements of the results that we need since some of the relevant papers are not readily available in English.

Definition 1. Let $\mu$ and $v$ be probability measures on $\mathbb{N}_{0}:=\mathbb{N} \cup\{0\}$ and $\mathbb{Z}$, respectively, and let $\xi_{i}^{(j)}$ and $\eta_{k}(i, j \geq 1, k \geq 0)$ be independent random variables such that each $\xi_{i}^{(j)}$ has distribution $\mu$ and each $\eta_{k}$ has distribution $v$. Then the process $\left(Z_{k}\right)_{k \geq 0}$, recursively defined by

$$
Z_{0}:=0, \quad Z_{k+1}:=\xi_{1}^{(k+1)}+\ldots+\xi_{Z_{k}+\eta_{k}}^{(k+1)}, \quad k \geq 0,
$$

is said to be a $(\mu, v)$-branching process with offspring distribution $\mu$ and migration distribution $v$. (Here we make an agreement that $\xi_{1}^{(k+1)}+\ldots+\xi_{i}^{(k+1)}=0$ if $i \leq 0$.) An offspring distribution $\mu$ which we shall use frequently is the geometric distribution with parameter $1 / 2$ and support $\mathbb{N}_{0}$. It is denoted by Geom(1/2) .

Note that any $(\mu, v)$-branching process is a time homogeneous Markov chain, whose distribution is determined by $\mu$ and $v$. More precisely, if at time $k$ the size of the population is $Z_{k}$ then (1) $\eta_{k}$ individuals immigrate or $\min \left\{Z_{k},\left|\eta_{k}\right|\right\}$ individuals emigrate depending on whether $\eta_{k} \geq 0$ or $\eta_{k}<0$ respectively, and (2) the resultant $\left(Z_{k}+\eta_{k}\right)_{+}$individuals reproduce independently according to the distribution $\mu$. This determines the size $Z_{k+1}$ of the population at time $k+1$.

In the current paper we are interested in the case when both the immigration and the emigration components are non-trivial and the number of emigrants is bounded from above. This bound will be the same as the bound $M$ on the number of cookies per site. We shall assume that

$$
v(\mathbb{N})>0 \quad \text { and } \quad v(\{k \in \mathbb{Z} \mid k \geq-M\})=1 .
$$

Denote the average migration by

$$
\lambda:=\sum_{k \geq-M} k v(\{k\})
$$

and the moment generating function of the offspring distribution by

$$
f(s):=\sum_{k \geq 0} \mu(\{k\}) s^{k}, \quad s \in[0,1] .
$$

In addition to (5), we shall make the following assumptions on the measures $\mu$ and $v$ : 
(A) $\quad f(0)>0, \quad f^{\prime}(1)=1, \quad b:=f^{\prime \prime}(1) / 2<\infty, \quad \lambda<\infty$;

(B) $\quad \sum_{k \geq 1} \mu(\{k\}) k^{2} \ln k<\infty$.

Note that $\mu=\operatorname{Geom}(1 / 2)$ satisfies condition (A) on the moment generating function $f$ with $b=1$. It also satisfies (B).

Next we state a result from the literature, which relates the limiting behavior of the process $\left(Z_{k}\right)_{k \geq 0}$ to the value of the parameter

$$
\theta:=\frac{\lambda}{b}
$$

At first, introduce the stopped process $\left(\widetilde{Z}_{k}\right)_{k \geq 0}$. Let

$$
N^{(Z)}:=\inf \left\{k \geq 1 \mid Z_{k}=0\right\} \quad \text { and } \quad \widetilde{Z}_{k}:=Z_{k} \mathbf{1}_{\left\{k<N^{(Z)}\right\}} .
$$

Note that the process $\left(\widetilde{Z}_{k}\right)_{k \geq 0}$ follows $\left(Z_{k}\right)_{k \geq 0}$ until the first time $\left(Z_{k}\right)_{k \geq 0}$ returns to 0 . Then $\left(\widetilde{Z}_{k}\right)_{k \geq 0}$ stays at 0 whereas $\left(Z_{k}\right)_{k \geq 0}$ eventually regenerates due to the presence of immigration (see the first inequality in (5)).

Theorem A ([FY89], [FYK90]). Let $\left(Z_{k}\right)_{k \geq 0}$ be a $(\mu, v)$-branching process satisfying (5), (A) and (B). We let

$$
u_{n}:=P\left[N^{(Z)}>n\right]=P\left[\widetilde{Z}_{n}>0\right], \quad n \in \mathbb{N},
$$

describe the tail of the distribution of $N^{(Z)}$ and denote by

$$
v_{n}:=E\left[\sum_{m=0}^{n} \widetilde{Z}_{m}\right]
$$

the expectation of the total progeny of $\left(\widetilde{Z}_{k}\right)_{k \geq 0}$ up to time $n \in \mathbb{N}_{0} \cup\{\infty\}$. Then the following statements hold.

(i) If $\theta>1$ then $\lim _{n \rightarrow \infty} u_{n}=c_{1} \in(0,1)$, in particular, the process $\left(\widetilde{Z}_{k}\right)_{k \geq 0}$ has a strictly positive chance $c_{1}$ never to die out.

(ii) If $\theta=1$ then $\lim _{n \rightarrow \infty} u_{n} \ln n=c_{2} \in(0, \infty)$, in particular, the process $\left(\widetilde{Z}_{k}\right)_{k \geq 0}$ will eventually die out a.s..

(iii) If $\theta=-1$ then $\lim _{n \rightarrow \infty} v_{n}(\ln n)^{-1}=c_{3} \in(0, \infty)$, in particular, $v_{\infty}=\infty$, i.e. the expected total progeny of $\left(\widetilde{Z}_{k}\right)_{k \geq 0}, v_{\infty}$, is infinite.

(iv) If $\theta<-1$ and

$$
\sum_{k \geq 1} k^{1+|\theta|} \mu(\{k\})<\infty
$$

then $\lim _{n \rightarrow \infty} u_{n} n^{1+|\theta|}=c_{4} \in(0, \infty)$. Moreover, in this case $\lim _{n \rightarrow \infty} v_{n}=c_{5} \in(0, \infty)$, i.e. the expected total progeny of $\left(\widetilde{Z}_{k}\right)_{k \geq 0}$ is finite. 
The above results about the limiting behavior of $u_{n}$ are contained in Theorems 1 and 4 of [FY89], [FYK90]. The proofs are given only in [FYK90]. The behavior of $v_{n}$ is the content of formula (33) in [FYK90]. The statements (i) and (ii) of Theorem A also follow from [YY95, Theorem 2.2] (see also [YMY03, Theorem 2.1]).

Remark 1. We have to point out that we use a slightly different (and more convenient for our purposes) definition of the lifetime, $N^{(Z)}$, of the stopped process. More precisely, our quantity $u_{n}$ can be obtained from the one in [FYK90] by the shift of the index from $n$ to $n-1$ and multiplication by

$$
P\left[\widetilde{Z}_{1}>0\right]=\sum_{k \geq 1} v(\{k\})\left(1-\mu(\{0\})^{k}\right),
$$

which is positive due to the first inequality in (5) and the fact that $\mu(\{0\})<1$ (by the condition $f^{\prime}(1)=1$ of assumption (A)). A similar change is needed for the expected total progeny of the stopped process. Clearly, these modifications affect only the values of constants in Theorem A and not their positivity or finiteness.

The papers mentioned above contain other results but we chose to state only those that we need. In fact, we only use the first part of (iv) and the following characterization, which we obtain from Theorem A by a coupling argument.

Corollary 4. Let the assumptions of Theorem A hold. Then $\left(\widetilde{Z}_{k}\right)_{k \geq 0}$ dies out a.s. iff $\theta \leq 1$. Moreover, the expected total progeny of $\left(\widetilde{Z}_{k}\right)_{k \geq 0}, v_{\infty}$, is finite iff $\theta<-1$.

Proof. Theorem A (i) gives the 'only if'-part of the first statement. To show the 'if' direction we assume that $\theta \leq 1$, i.e. $v$ has mean $\lambda \leq b$ (see (7)). Then there is another $v^{\prime}$ with mean $b$ which stochastically dominates $v$. Indeed, if $X$ has distribution $v$ and $Y$ has expectation $b-\lambda$ and takes values in $\mathbb{N}_{0}$ then $v^{\prime}$ can be chosen as the distribution of $X+Y$. By coupling, the $\left(\mu, v^{\prime}\right)$-branching process stochastically dominates the $(\mu, v)$-branching process. However, the $\left(\mu, v^{\prime}\right)$-branching process dies out a.s. due to Theorem A (ii) since for this process $\theta=1$. Consequently, the $(\mu, v)$-branching process must die out, too.

Similarly, Theorem A (iv) gives the 'if'-part of the second statement. The converse direction follows from monotonicity as above and Theorem A (iii).

\section{From ERWs to branching processes with migration}

The goal of this mainly expository section is to show how our ERW model can be naturally recast as a branching process with migration. This connection was already observed and used in [BS08a] and $[\mathrm{BSO} 0 \mathrm{~b}]$.

Consider a nearest neighbor random walk path $\left(X_{n}\right)_{n \geq 0}$, which starts at 0 and define

$$
T_{k}:=\inf \left\{n \geq 1 \mid X_{n}=k\right\} \in \mathbb{N} \cup\{\infty\}, \quad k \in \mathbb{Z} .
$$

Assume for the moment that $X_{1}=1$ and consider the right excursion, i.e. $\left(X_{n}\right)_{0 \leq n<T_{0}}$. The left excursion can be treated by symmetry.

On the set $\left\{T_{0}<\infty\right\}$ we can define a bijective path-wise mapping of this right excursion to a finite rooted tree, which corresponds to a realization of a branching process with the extinction time 


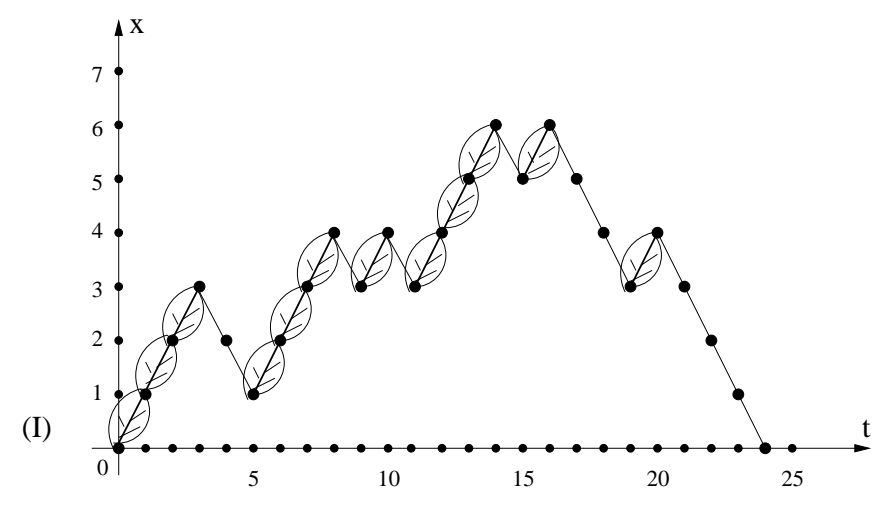

(II)

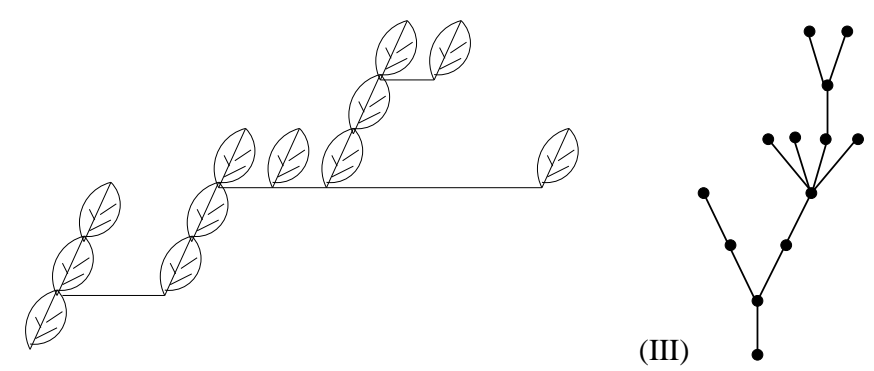

Figure 2: (I) Right excursion of the random walk. Upcrossings are marked by "tree leaves". (II) The number of upcrossings of the edge $(k, k+1)$ becomes the number of particles in generation $k$ for the branching process. Shrinking the horizontal lines in (II) into single points gives the tree (III). Traversing the tree (III) in preorder rebuilds the excursion (I).

$\max \left\{X_{n}, 0 \leq n<T_{0}\right\}$ as illustrated in Figure 2. Moreover, given a tree for a branching process that becomes extinct in finite time, we can reconstruct the right excursion of the random walk. This can be done by making a time diagram of up and down movements of an ant traversing the tree in preorder: the ant starts at the root, always chooses to go up and to the left whenever possible, never returns to an edge that was already crossed in both directions, and finishes the journey at the root (Figure 2, (III)).

The above path-wise correspondence on $\left\{T_{0}<\infty\right\}$ does not depend on the measure associated to the random walk paths. To consider the set $\left\{T_{0}=\infty\right\}$ we shall need some of the properties of this measure. The following simple statement leaves only three major possibilities for a long term behavior of an ERW path.

Lemma 5. Let $\omega \in \Omega_{M}$. Then $P_{0, \omega}$ a.s.

$$
\liminf _{n \rightarrow \infty} X_{n}, \limsup _{n \rightarrow \infty} X_{n} \in\{-\infty,+\infty\}
$$

Proof. Let $z \in \mathbb{Z}$. If the ERW visits $z$ infinitely many times then it also visits $z+1$ infinitely many times due to the second Borel Cantelli lemma, the strong Markov property, and the assumption $\omega(z, i)=1 / 2$ for $i>M$. This implies $P_{0, \omega}$-a.s. $\lim \sup _{n} X_{n} \notin \mathbb{Z}$. Similarly, $P_{0, \omega}$-a.s. $\liminf _{n} X_{n} \notin$ $\mathbb{Z}$.

Let us now put a measure on the paths and see what kind of measure will be induced on trees. Consider the right excursion of the simple symmetric random walk. Assume without loss of generality 
that the walk starts at 1 . Then the probability that $T_{0}<\infty$ is equal to one and the corresponding measure on trees will be the one for a standard Galton-Watson process with the Geom(1/2) offspring distribution starting from a single particle. More precisely, set $U_{0}:=1$ and let

$$
U_{k}:=\#\left\{n \geq 0 \mid n<T_{0}, X_{n}=k, X_{n+1}=k+1\right\}, \quad k \geq 1,
$$

be the number of upcrossings of the edge $(k, k+1)$ by the walk before it hits 0 . Then $\left(U_{k}\right)_{k \geq 0}$ has the same distribution as the Galton-Watson process with Geom(1/2) offspring distribution. Therefore, $\left(U_{k}\right)_{k \geq 0}$ can also be generated as follows: start with one particle: $U_{0}=1$. To generate the $(k+1)$ st generation from the $k$-th generation (assuming that the process has not yet died out), the first particle of generation $k$ tosses a fair coin repeatedly and produces one offspring if the coin comes up "heads". It stops the reproduction once the coin comes up "tails". Then the second particle in generation $k$ follows the same procedure independently, then the third one, and so on. Consequently,

$$
U_{k+1}=\xi_{1}^{(k+1)}+\ldots+\xi_{U_{k}}^{(k+1)}
$$

where $\xi_{i}^{(j)}, i, j \geq 1$ are independent with distribution $\operatorname{Geom}(1 / 2)$.

To construct a branching process corresponding to an ERW with $M$ cookies per site one can use exactly the same procedure except that for the first $M$ coin tosses in the $k$-th generation the particles should use coins with biases "prescribed" by the cookies located at site $k$. Since every particle tosses a coin at least once, at most the first $M$ particles in each generation will have a chance to use biased coins. All the remaining particles will toss fair coins only. This can be viewed as a branching process with migration in the following natural way. Before the reproduction starts, the first $U_{k} \wedge M$ particles emigrate, taking with them all $M$ biased coins and an infinite supply of fair coins. In exile they reproduce according to the procedure described above. Denote the total number of offspring produced by these particles by $\eta_{U_{k} \wedge M}^{(k+1)}$. Meanwhile, the remaining particles (if any) reproduce using only fair coins. Finally, the offspring of the emigrants re-immigrate. Therefore, the number of particles in the generation $k+1$ can be written as

$$
U_{k+1}:=\xi_{1}^{(k+1)}+\ldots+\xi_{U_{k}-M}^{(k+1)}+\eta_{U_{k} \wedge M}^{(k+1)}
$$

where $\xi_{i}^{(j)}$ and $\eta_{\ell}^{(k)}(i, j, k \geq 1,0 \leq \ell \leq M)$ are independent random variables, each one of the sequences $\left(\eta_{0}^{(k)}\right)_{k \geq 1}, \ldots,\left(\eta_{M}^{(k)}\right)_{k \geq 1}$ is identically distributed, and each $\xi_{i}^{(j)}$ has distribution Geom(1/2)

Branching processes of type (10) were considered in [BS08a] (p. 630) and [BS08b] (p. 815), except that they were generated not by the forward but by the backward excursion (see (29) in Section 6). Careful analysis of such processes carried out in these two papers yielded results concerning positive speed and rates of growth at infinity for ERWs with non-negative cookies. However, from a practical point of view, $(\mu, v)$-branching processes, introduced in Definition 1, seem to be well-known and studied more extensively in the past. In particular, we could find the results we need (see Theorem A) in the literature only for $(\mu, v)$-branching processes, but not for processes of the form (10). For this reason one of our main tasks will be to relate these two classes of processes in order to translate the results from the literature into results about processes of the form (10). 


\section{Coin-toss construction of the ERW and the related $(\mu, v)$-branching process}

In this section we formalize a coin-toss construction of the ERW and introduce auxiliary processes used in the rest of the paper.

Let $(\Omega, \mathscr{F})$ be some measurable space equipped with a family of probability measures $P_{x, \omega}, x \in$ $\mathbb{Z}, \omega \in \Omega_{M}$, such that for each choice of $x \in \mathbb{Z}$ and $\omega \in \Omega_{M}$ we have \pm 1 -valued random variables $Y_{i}^{(k)}, k \in \mathbb{Z}, i \geq 1$, which are independent under $P_{x, \omega}$ with distribution given by

$$
P_{x, \omega}\left[Y_{i}^{(k)}=1\right]=\omega(k, i) \quad \text { and } \quad P_{x, \omega}\left[Y_{i}^{(k)}=-1\right]=1-\omega(k, i) .
$$

Moreover, we require that there is a random variable $X_{0}$ on $\left(\Omega, \mathscr{F}, P_{x, \omega}\right)$ such that $P_{x, \omega}\left[X_{0}=x\right]=1$. Then an ERW $\left(X_{n}\right)_{n \geq 0}$, starting at $x \in \mathbb{Z}$, in the environment $\omega$ can be realized on the probability space $\left(\Omega, \mathscr{F}, P_{x, \omega}\right)$ recursively by:

$$
X_{n+1}:=X_{n}+Y_{\#\left\{i \leq n \mid X_{i}=X_{n}\right\}}^{\left(X_{n}\right)}, \quad n \geq 0 .
$$

We shall refer to $\left\{Y_{i}^{(k)}=1\right\}$ as a "success" and to $\left\{Y_{i}^{(k)}=-1\right\}$ as a "failure". Due to (11) every step to the right or to the left of the random walk corresponds to a success or a failure, respectively.

We now describe various branching processes that appear in the proofs. Namely, we introduce processes $\left(V_{k}\right)_{k \geq 0},\left(W_{k}\right)_{k \geq 0}$, and $\left(Z_{k}\right)_{k \geq 0}$. Modifications of the first two processes suitable for left excursions will be defined later when they are needed (we shall keep the same notation though, hoping that this will not lead to confusion). The last process, $\left(Z_{k}\right)_{k \geq 0}$, will belong to the class of processes from Section 2.

For $m \in \mathbb{N}$ and $k \in \mathbb{Z}$ let

$$
S_{0}^{(k)}:=0, \quad S_{m}^{(k)}:=\# \text { of successes in }\left(Y_{i}^{(k)}\right)_{i \geq 1} \text { prior to the } m \text {-th failure. }
$$

Recall from the introduction that $P_{x}[\cdot]$ denotes the averaged measure $\mathbb{E}\left[P_{x, \omega}[\cdot]\right]$. By assumption (2) the walk reaches 1 in one step with positive $P_{0}$-probability.

We shall be interested in the behavior of the process $\left(U_{k}\right)_{k \geq 0}$ defined in (9). At first, we shall relate $\left(U_{k}\right)_{k \geq 0}$ to $\left(V_{k}\right)_{k \geq 0}$ which is recursively defined by

$$
V_{0}:=1, \quad V_{k+1}:=S_{V_{k}}^{(k+1)}, \quad k \geq 0 .
$$

Observe that $\left(V_{k}\right)_{k \geq 0}$ is a time homogeneous Markov chain, as the sequence of sequences $\left(S_{m}^{(k)}\right)_{m \geq 0}$, $k \geq 0$, is i.i.d.. Moreover, 0 is an absorbing state for $\left(V_{k}\right)_{k \geq 0}$. We claim that under $P_{1}$,

$$
\begin{aligned}
& U_{k}=V_{k} \quad \text { for all } k \geq 0 \text { on the event }\left\{T_{0}<\infty\right\} \\
& U_{k} \leq V_{k} \quad \text { for all } k \geq 0 \text { on the event }\left\{T_{0}=\infty\right\} .
\end{aligned}
$$

The relation (14) is obvious from the discussion in Section 3 and Figure 2. To show (15) we shall use induction. Recall that $U_{0}=V_{0}=1$ and assume $U_{i} \leq V_{i}$ for all $i \leq k$. From Lemma 5 we know that $X_{n} \rightarrow \infty$ as $n \rightarrow \infty$ on $\left\{T_{0}=\infty\right\}$ a.s. with respect to $P_{1}$. Therefore, the last, $U_{k}$-th, upcrossing of the edge $(k, k+1)$ by the walk is not matched by a downcrossing. This implies that $U_{k+1}$ should be 
less than or equal to the number of successes in the sequence $\left(Y_{i}^{(k+1)}\right)_{i \geq 1}$ prior to the $U_{k}$-th failure. On the other hand, to get the value of $V_{k+1}$ one needs to count all successes in this sequence until the $V_{k}$-th failure. Since $U_{k} \leq V_{k}$, we conclude that $U_{k+1} \leq V_{k+1}$.

Next we introduce the process $\left(W_{k}\right)_{k \geq 0}$ by setting

$$
W_{0}:=0, \quad W_{k+1}:=S_{W_{k} \vee M}^{(k)}, \quad k \geq 0 .
$$

Just as $\left(V_{k}\right)_{k \geq 0}$, the process $\left(W_{k}\right)_{k \geq 0}$ is a time homogeneous Markov chain on non-negative integers. Moreover, the transition probabilities from $i$ to $j$ of these two processes coincide except for $i \in\{0,1, \ldots, M-1\}$ and both processes can reach any positive number with positive probability. Therefore, if one of these two processes goes to infinity with positive probability, so does the other:

$$
P_{1}\left[V_{k} \rightarrow \infty\right]>0 \quad \Longleftrightarrow \quad P_{1}\left[W_{k} \rightarrow \infty\right]>0
$$

Finally, we decompose the process $\left(W_{k}\right)_{k \geq 0}$ into two components as follows.

Lemma 6. For $k \geq 0$ let $Z_{k}:=W_{k+1}-S_{M}^{(k)}$. Then $\left(Z_{k}\right)_{k \geq 0}$ is a (Geom(1/2), $\left.v\right)$-branching process, where $v$ is the common distribution of $\eta_{k}:=S_{M}^{(k)}-M$ under $P_{1}$.

Proof. By definition, $Z_{0}=0$ and

$$
Z_{k+1}=W_{k+2}-S_{M}^{(k+1)} \stackrel{(16)}{=} S_{W_{k+1} \vee M}^{(k+1)}-S_{M}^{(k+1)}=\xi_{1}^{(k+1)}+\cdots+\xi_{W_{k+1}-M}^{(k+1)},
$$

where $\xi_{i}^{(k+1)}$ is defined as the number of successes in $\left(Y_{j}^{(k+1)}\right)_{j \geq 1}$ between the $(M+i-1)$-th and the $(M+i)$-th failure, $i \geq 1$. Therefore, by definition of $Z_{k}$ and $\eta_{k}$,

$$
Z_{k+1}=\xi_{1}^{(k+1)}+\cdots+\xi_{Z_{k}+S_{M}^{(k)}-M}^{(k+1)}=\xi_{1}^{(k+1)}+\cdots+\xi_{Z_{k}+\eta_{k}}^{(k+1)}
$$

Since $\omega(k, m)=1 / 2$ for $m>M$, the random variables $Y_{m}^{(k)}, m>M, k \geq 0$, are independent and uniformly distributed on $\{-1,1\}$ under $P_{1}$. From this we conclude that the $\xi_{i}^{(k)}, k, i \geq 1$, have distribution $\operatorname{Geom}(1 / 2)$. To show the independence of $\xi_{i}^{(j)}$ and $\eta_{k}(i, j \geq 1, k \geq 0)$, as required by Definition 1 , notice that $\eta_{k}=S_{M}^{(k)}-M$ depends only on $Y_{m}^{(k)}$, where $m$ changes from 1 to the number of the trial resulting in the $M$-th failure inclusively, while each $\xi_{i}^{(k)}, i \geq 1$, counts the number of successes in $\left(Y_{m}^{(k)}\right)_{m \geq 1}$ between the $(M+i-1)$-th and the $(M+i)$-th failure. Recalling again that $Y_{m}^{(k)}, m \geq 1, k \geq 0$, are independent under $P_{1}$, we get the desired independence.

Having introduced all necessary processes we can now turn to the proofs of our results.

\section{Recurrence and transience}

Definition 2. The ERW is called recurrent from the right if the first excursion to the right of 0 , if there is any, is $P_{0}$-a.s. finite. Recurrence from the left is defined analogously. 
In the next lemma we shall characterize ERW which are recurrent from the right in terms of branching processes with migration. At first, we shall introduce a relaxation of condition (1), which is needed for the proof of Theorem 1 :

The sequence $(\omega(k, \cdot))_{k \geq K}$ is i.i.d. under $\mathbb{P}$ for some $K \in \mathbb{N}$.

Under this assumption the sequence indexed by $k \geq K$ of sequences $\left(Y_{i}^{(k)}\right)_{i \geq 1}$ is i.i.d. with respect to $P_{0}$. In particular, the sequence $\left(S_{M}^{(k)}\right)_{k \geq K}$ is i.i.d. under $P_{0}$.

Lemma 7. Replace assumption (1) by (18) and assumption (2) by

$$
\mathbb{E}\left[\prod_{i=1}^{M}(1-\omega(K, i))\right]>0 .
$$

Denote the common distribution of $\eta_{k}:=S_{M}^{(k)}-M, k \geq K$, under $P_{0}$ by $v$. Then the ERW is recurrent from the right if and only if the (Geom(1/2), $v)$-branching process dies out a.s., i.e. reaches state 0 at some time $k \geq 1$.

Proof. Since we are interested in the first excursion to the right we may assume without loss of generality that the random walk starts at 1 . Then, recalling definition (9), we have $\left\{T_{0}=\infty\right\} \stackrel{P_{1}}{=}$ $\left\{\forall k \geq 1 U_{k}>0\right\}$, where $A \stackrel{P_{1}}{=} B$ means that the two events $A$ and $B$ may differ by a $P_{1}$-null-set only. Indeed, since $U_{k}$ counts only upcrossings of the edge $(k, k+1)$ prior to $T_{0}$, the inclusion $\supseteq$ is trivial. The reverse relation follows from Lemma 5 . This together with (14) and (15) implies that

$$
\left\{T_{0}=\infty\right\} \stackrel{P_{1}}{=}\left\{\forall k \geq 0 V_{k}>0\right\} .
$$

As above $\left(V_{k}\right)_{k \geq K}$ is a time homogeneous Markov chain since the sequence of sequences $\left(S_{m}^{(k)}\right)_{m \geq 0}$, $k \geq K$, is i.i.d.. For any $m$ the transition probability of this Markov chain from $m \in \mathbb{N}$ to 0 is equal to

$$
P_{1}\left[S_{m}^{(K)}=0\right]=\mathbb{E}\left[\prod_{i=1}^{m}(1-\omega(K, i))\right],
$$

which is strictly positive by (19). Since 0 is absorbing for $\left(V_{k}\right)_{k \geq 0}$ we get that $\left\{\forall k \geq 0 V_{k}>0\right\} \stackrel{P_{1}}{=}$ $\left\{V_{k} \rightarrow \infty\right\}$. Consequently, by (20), $\left\{T_{0}=\infty\right\} \stackrel{P_{1}}{=}\left\{V_{k} \rightarrow \infty\right\}$. Next we turn to the process $\left(W_{k}\right)_{k \geq 0}$ and recall relation (17). Thus,

$$
P_{1}\left[T_{0}=\infty\right]=0 \quad \Longleftrightarrow \quad P_{1}\left[W_{k} \rightarrow \infty\right]=0 .
$$

Finally, we decompose the process $\left(W_{k}\right)_{k \geq 0}$ as in Lemma 6 by writing $W_{k+1}=Z_{k}+S_{M}^{(k)}$ for $k \geq 0$, where $\left(Z_{k}\right)_{k \geq K}$ is a Markov chain with the transition kernel of a $(\mathrm{Geom}(1 / 2), v)$-branching process. Since the sequence $\left(S_{M}^{(k)}\right)_{k \geq K}$ is i.i.d., this implies that $\left\{W_{k} \rightarrow \infty\right\} \stackrel{P_{1}}{=}\left\{Z_{k} \rightarrow \infty\right\}$. Together with (21) this shows that the ERW is recurrent from the right iff $P_{0}\left[Z_{k} \rightarrow \infty\right]=0$. Since $\left(Z_{k}\right)_{k \geq K}$ is an irreducible Markov chain this is equivalent to $\left(Z_{k}\right)_{k \geq K}$ being recurrent, which is equivalent to recurrence of the state 0 for $(\operatorname{Geom}(1 / 2), v)$-branching processes. 
Lemma 8. Assume again (1) and (2). If the ERW is recurrent from the right then all excursions to the right of $O$ are $P_{0}$-a.s. finite. If the ERW is not recurrent from the right then it will make $P_{0}$-a.s. only a finite number of excursions to the right. The corresponding statements hold for recurrence from the left.

Proof. Let the ERW be recurrent from the right. By Definition 2 the first excursion to the right is a.s. finite. By Lemma 7 the corresponding (Geom(1/2), $v$ )-branching process dies out a.s.. Let $i \geq 1$ and assume that all excursions to the right up to the $i$-th one have been proven to be $P_{0}$-a.s. finite. If the ERW starts the $(i+1)$-st excursion to the right of 0 then it finds itself in an environment which has been modified by the previous $i$ excursions up to a random level $R \geq 1$, beyond which the environment has not been touched yet. Therefore, conditioning on the event $\{R=K\}, K \geq 1$, puts us within the assumptions of Lemma 7: the random walk starts the right excursion from 0 in a random cookie environment which satisfies (18). But the corresponding (Geom(1/2), $v$ )-branching process is still the same and, thus, dies out a.s.. Therefore, this excursion, which is the $(i+1)$-st excursion of the walk, is a.s. finite on $\{R=K\}$. Since by our induction assumption the events $\{R=K\}, K \geq 1$, form a partition of a set of full measure, we obtain the first statement of the lemma.

For the second statement let

$$
D:=\inf \left\{n \geq 1 \mid X_{n}<X_{0}\right\}
$$

be the first time that the walk backtracks below its starting point. Due to (2), $P_{0}\left[X_{1}=1\right]>0$. Therefore, since the walk is assumed to be not recurrent from the right,

$$
P_{0}[D=\infty]>0
$$

Denote by $K_{i}$ the right-most visited site before the end of the $i$-th excursion and define $K_{i}=\infty$ if there is no $i$-th right excursion or if the $i$-th excursion to the right covers $\mathbb{N}$. Then the number of $i \geq 1$ such that $K_{i}<K_{i+1}$, is stochastically bounded from above by a geometric distribution with parameter $P_{0}[D=\infty]$. Indeed, each time the walk reaches a level $K_{i}+1<\infty$, which it has never visited before, it has probability $P_{0}[D=\infty]$ never to backtrack again below the level $K_{i}+1$, independently of its past. Therefore, $\left(K_{i}\right)_{i}$ increases only a finite number of times. Hence $P_{0}$-a.s. $R:=\sup \left\{K_{i} \mid i \geq 1, K_{i}<\infty\right\}<\infty$. Now, if the walk did an infinite number of excursions to the right, then, $P_{0}$-a.s. $\sup _{n} X_{n}=R<\infty$ and $\limsup _{n} X_{n} \geq 0$, which is impossible due to Lemma 5 .

Proposition 9. The ERW is recurrent from the right if and only if $\delta \leq 1$. Similarly, it is recurrent from the left if and only if $\delta \geq-1$.

For the proof we need the next lemma, which relates the parameter $\delta$ of the ERW and the parameter $\theta$ of the branching process with migration.

Lemma 10. Let $v$ be the distribution of $S_{M}^{(0)}-M$ under $P_{0}$. Then $\theta$ defined in (7) for the $(\operatorname{Geom}(1 / 2), v)$-branching process is equal to $\delta$ defined in (3).

Proof of Lemma 10. For $\mu=\operatorname{Geom}(1 / 2)$ the parameter $b$ defined in (A) equals 1 . Hence, by (6), $\theta=\lambda=E_{0}\left[S_{M}^{(0)}-M\right]$. Thus it suffices to show that

$$
E_{0}\left[S_{M}^{(0)}\right]-M=\delta
$$

This has already been observed in [BS08b, Lemma 3.3]. For completeness, we include a proof. Let $F:=\#\left\{1 \leq i \leq M \mid Y_{i}^{(0)}=-1\right\}$ be the number of failures among the first $M$ trials. Then $M-F$ is the 
number of successes among the first $M$ trials. Therefore, since $S_{M}^{(0)}$ is the total number of successes prior to the $M$-th failure, $S_{M}^{(0)}-(M-F)$ is the number of successes after the $M$-th trial and before the $M$-th failure. Given $F$, its distribution is negative binomial with parameters $M-F$ and $p=1 / 2$, i.e. the $(M-F)$-fold convolution of Geom(1/2), and therefore has mean $M-F$. Thus,

$$
E_{0}\left[S_{M}^{(0)}-(M-F)\right]=E_{0}\left[E_{0}\left[S_{M}^{(0)}-(M-F) \mid F\right]\right]=E_{0}[M-F] .
$$

Subtracting $E_{0}[F]$ from both sides we obtain

$$
E_{0}\left[S_{M}^{(0)}\right]-M=M-2 \sum_{i=1}^{M} \mathbb{E}[1-\omega(0, i)]=\sum_{i=1}^{M}(2 \mathbb{E}[\omega(0, i)]-1)=\delta .
$$

Proof of Proposition 9. Due to Lemma 7 the walk is recurrent from the right iff the (Geom(1/2), v)branching process dies out a.s., where $v$ is the distribution of $S_{M}^{(0)}-M$. By the first statement of Corollary 4 this is the case iff $\theta \leq 1$. The first claim of the proposition follows now from Lemma 10 . The second one follows by symmetry.

Proof of Theorem 1. If $\delta>1$ then by Proposition 9 the walk is not recurrent from the right but recurrent from the left. If the walk returned infinitely often to 0 then it would also make an infinite number of excursions to the right which is impossible due to Lemma 8. Hence the ERW visits 0 only finitely often. Since any left excursion is finite due to Lemma 8 the last excursion is to the right and is infinite. Consequently, $P_{0}$-a.s. $\liminf _{n} X_{n} \geq 0$, and therefore, due to Lemma $5, X_{n} \rightarrow \infty$. Similarly, $\delta<-1$ implies $P_{0}$-a.s. $X_{n} \rightarrow \infty$.

In the remaining case $\delta \in[-1,1]$ all excursions from 0 are finite due to Proposition 9. Hence, 0 is visited infinitely many times.

Remark 2. The equivalence (20) also holds correspondingly for one-dimensional random walks $\left(X_{n}\right)_{n \geq 0}$ in i.i.d. random environments (RWRE) and branching processes $\left(V_{k}\right)_{k \geq 0}$ in random environments, i.e. whose offspring distribution is geometric with a random parameter. This way the recurrence theorem due to Solomon [So75, Th. (1.7)] for RWRE can be deduced from results by Athreya and Karlin, see [AN72, Chapter VI.5, Corollary 1 and Theorem 3].

\section{A renewal structure for transient ERW}

A powerful tool for the study of random walks in random environments (RWRE) is the so-called renewal or regeneration structure. It is already present in [KKS75], [Ke77] and was first used for multi-dimensional RWRE in [SZ99]. It has been mentioned in [Zer05, p. 114, Remark 3] that this renewal structure can be straightforwardly adapted to the setting of directionally transient ERW in i.i.d. environments in order to give a law of large numbers. The proofs of positivity of speed and of a central limit theorem for once-excited random walks in dimension $d \geq 2$ in [BR07] were also phrased in terms of this renewal structure. We shall do the same for the present model.

We continue to assume (1) and (2). Let $\delta>1$, where $\delta$ is the average drift defined in (3). This means, due to Theorem 1, that $P_{0}$-a.s. $X_{n} \rightarrow \infty$. Moreover, by Proposition 9 , the walk is not recurrent 


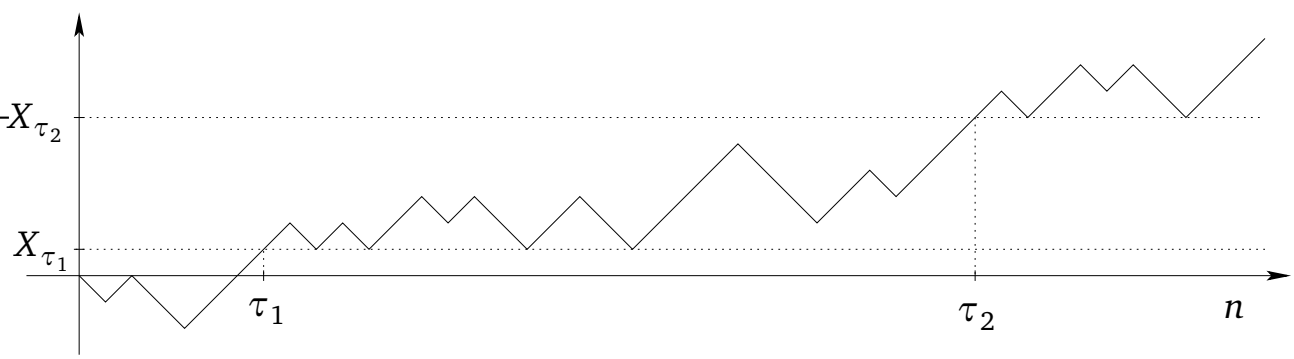

Figure 3: A random walk path with two renewals.

from the right, which implies, as we already mentioned, see (22), that $P_{0}[D=\infty]>0$. Hence there are $P_{0}$-a.s. infinitely many random times $n$, so-called renewal or regeneration times, with the defining property that $X_{m}<X_{n}$ for all $0 \leq m<n$ and $X_{m} \geq X_{n}$ for all $m>n$. Call the increasing enumeration of these times $\left(\tau_{k}\right)_{k \geq 1}$, see also Figure 3 . Then the sequence $\left(X_{\tau_{1}}, \tau_{1}\right),\left(X_{\tau_{k+1}}-X_{\tau_{k}}, \tau_{k+1}-\tau_{k}\right)(k \geq 1)$ of random vectors is independent under $P_{0}$. Furthermore, the random vectors $\left(X_{\tau_{k+1}}-X_{\tau_{k}}, \tau_{k+1}-\right.$ $\left.\tau_{k}\right), k \geq 1$, have the same distribution under $P_{0}$. For multidimensional RWRE and once-excited random walk the corresponding statement is [SZ99, Corollary 1.5] and [BR07, Proposition 3], respectively. It follows from the renewal theorem, see e.g. [Zei04, Lemma 3.2.5], that

$$
E_{0}\left[X_{\tau_{2}}-X_{\tau_{1}}\right]=P_{0}[D=\infty]^{-1}<\infty .
$$

Moreover, the ordinary strong law of large numbers implies that

$$
\lim _{n \rightarrow \infty} \frac{X_{n}}{n}=\frac{E_{0}\left[X_{\tau_{2}}-X_{\tau_{1}}\right]}{E_{0}\left[\tau_{2}-\tau_{1}\right]}=: v \quad P_{0} \text {-a.s., }
$$

see [SZ99, Proposition 2.1] and [Zei04, Theorem 3.2.2] for RWRE and also [BR07, Theorem 2] for once-ERW. Therefore,

$$
v>0 \quad \text { if and only if } \quad E_{0}\left[\tau_{2}-\tau_{1}\right]<\infty .
$$

If, moreover,

$$
E_{0}\left[\left(\tau_{2}-\tau_{1}\right)^{2}\right]<\infty
$$

then the result claimed in Theorem 3 holds with

$$
\sigma^{2}:=\frac{E_{0}\left[\left(X_{\tau_{2}}-X_{\tau_{1}}-v\left(\tau_{2}-\tau_{1}\right)\right)^{2}\right]}{E_{0}\left[\tau_{2}-\tau_{1}\right]}>0
$$

see [Sz00, Theorem 4.1] for RWRE and [BR07, Theorem 3 and Remark 1] for once-ERW.

Thus, in order to prove Theorems 2 and 3 we need to control the first and the second moment, respectively, of $\tau_{2}-\tau_{1}$. We start by introducing for $k \geq 0$ the number

$$
D_{k}:=\#\left\{n \mid \tau_{1}<n<\tau_{2}, X_{n}=X_{\tau_{2}}-k, X_{n+1}=X_{\tau_{2}}-k-1\right\}
$$

of downcrossings of the edge $\left(X_{\tau_{2}}-k, X_{\tau_{2}}-k-1\right)$ between the times $\tau_{1}$ and $\tau_{2}$.

Lemma 11. Assume that the ERW is transient to the right and let $p \geq 1$. Then the $p$-th moment of $\tau_{2}-\tau_{1}$ under $P_{0}$ is finite if and only if the $p$-th moment of $\sum_{k \geq 1} D_{k}$ is finite. 


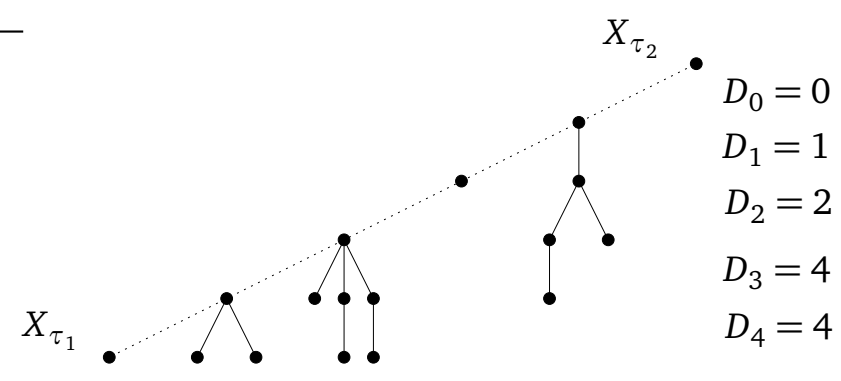

Figure 4: For the path in Figure 3 the process $\left(D_{k}\right)_{k \geq 0}$ is realized as $(0,1,2,4,4,0,0, \ldots)$. The solid lines represent downcrossings. The thick dots on the dashed line correspond to the single immigrant in definition (32).

Proof. The number of upcrossings between $\tau_{1}$ and $\tau_{2}$ is $X_{\tau_{2}}-X_{\tau_{1}}+\sum_{k \geq 1} D_{k}$, since $X_{\tau_{1}}<X_{\tau_{2}}$ and since each downcrossing needs to be balanced by an upcrossing. Each step is either an upcrossing or a downcrossing, therefore,

$$
\tau_{2}-\tau_{1}=X_{\tau_{2}}-X_{\tau_{1}}+2 \sum_{k \geq 1} D_{k}
$$

For every $k \in\left\{X_{\tau_{1}}+1, \ldots, X_{\tau_{2}}-1\right\}$ there is a downcrossing of the edge $(k, k-1)$, otherwise $k$ would be another point of renewal. Hence, $X_{\tau_{2}}-X_{\tau_{1}} \leq 1+\sum_{k \geq 1} D_{k}$ and, by (30),

$$
2 \sum_{k \geq 1} D_{k} \leq \tau_{2}-\tau_{1} \leq 1+3 \sum_{k \geq 1} D_{k}
$$

This implies the claim.

To interpret $\left(D_{k}\right)_{k \geq 0}$ as a branching process (see Figure 4) we define for $m \in \mathbb{N}$ and $k \in \mathbb{Z}$

$$
F_{0}^{(k)}:=0, \quad F_{m}^{(k)}:=\# \text { of failures in }\left(Y_{i}^{(k)}\right)_{i \geq 1} \text { prior to the } m \text {-th success. }
$$

(Compare this to the definition of $S_{m}^{(k)}$ in (12).) Let

$$
\begin{aligned}
& V_{0}:=0, \quad V_{k+1}:=F_{V_{k}+1}^{(k)}, \quad k \geq 0 ; \\
& \widetilde{V}_{k}:=V_{k} \mathbf{1}_{\left\{k<N^{(V)}\right\}}, \quad \text { where } \quad N^{(V)}:=\inf \left\{k \geq 1 \mid V_{k}=0\right\} .
\end{aligned}
$$

Lemma 12. Assume that the ERW is transient to the right. Then $\left(D_{k}\right)_{k \geq 0}$ and $\left(\widetilde{V}_{k}\right)_{k \geq 0}$ have the same distribution under $P_{0}$.

Proof. Fix an integer $K \geq 1$. For brevity, we set $\vec{D}:=\left(D_{1}, \ldots, D_{K}\right)$ and $\vec{V}:=\left(\widetilde{V}_{1}, \ldots, \widetilde{V}_{K}\right)$. It suffices to show that

$$
P_{0}[\vec{D}=\vec{i}]=P_{0}[\vec{V}=\vec{i}]
$$

for all $\vec{i} \in \mathbb{N}_{0}^{K}$. Since both processes start from 0 and also stay at 0 once they have returned to 0 for the first time, it is enough to consider vectors $\vec{i}$ whose entries are strictly positive except for maybe the last one. And, since the process $\left(D_{k}\right)_{k \geq 0}$ eventually does reach $0 P_{0}$-a.s., namely at $k=X_{\tau_{2}}-X_{\tau_{1}}<\infty$, it suffices to consider only $\vec{i}$ whose last entry is 0 . Thus, let $\vec{i}=\left(i_{1}, \ldots, i_{K}\right) \in \mathbb{N}_{0}^{K}$ with $i_{1}, \ldots, i_{K-1} \geq 1$ and $i_{K}=0$. At first, we shall show that

$$
P_{0}[\vec{D}=\vec{i}]=P_{0}\left[\vec{D}^{(K)}=\vec{i}\right] \text {, }
$$


where, for $m, k \geq 0$,

$$
\begin{aligned}
D_{k}^{(m)} & :=\#\left\{n<T_{m} \mid X_{n}=m-k, X_{n+1}=m-k-1\right\} \text { and } \\
\vec{D}^{(m)} & :=\left(D_{1}^{(m)}, \ldots, D_{K}^{(m)}\right) .
\end{aligned}
$$

We start from the partition equation

$$
P_{0}[\vec{D}=\vec{i}]=\sum_{m \geq 0} P_{0}\left[\vec{D}=\vec{i}, \tau_{2}=T_{m}\right] .
$$

However, on the event $\left\{\vec{D}=\vec{i}, \tau_{2}=T_{m}\right\}$, we have $X_{\tau_{1}}=m-K$ by our choice of $\vec{i}$. Since $X_{\tau_{1}} \geq 0$, we may start the summation in (37) from $m=K$. Moreover, comparing the definitions (29) and (36), we see that $\left\{\vec{D}=\vec{i}, \tau_{2}=T_{m}\right\}=\left\{\vec{D}^{(m)}=\vec{i}, \tau_{2}=T_{m}\right\}$, using that not only on the left but also on the right event we have $X_{\tau_{1}}=m-K$. Hence, the right hand side of (37) is equal to

$$
\sum_{m \geq K} P_{0}\left[\vec{D}^{(m)}=\vec{i}, \tau_{2}=T_{m}\right]
$$

Notice that the event $\left\{\tau_{2}=T_{m}\right\}$ occurs if and only if the ERW does not fall below level $m$ after time $T_{m}$ and if exactly one of the numbers $D_{1}^{(m)}, \ldots, D_{m}^{(m)}$ is equal to 0 . Then, by our choice of $\vec{i}$, (38) is equal to

$$
\sum_{m \geq K} P_{0}\left[\vec{D}^{(m)}=\vec{i}, \forall k=K+1, \ldots, m: D_{k}^{(m)} \geq 1, \forall n \geq T_{m}: X_{n} \geq m\right] .
$$

By the strong Markov property applied to the stopping time $T_{m}$ and by independence in the environment this equals

$$
\sum_{m \geq K} P_{0}\left[\vec{D}^{(m)}=\vec{i}, \forall k=K+1, \ldots, m: D_{k}^{(m)} \geq 1\right] P_{m}[D=\infty] .
$$

Since $i_{K}=0$, this is the same as

$$
\sum_{m \geq K} P_{0}\left[\vec{D}^{(m)}=\vec{i}, \forall k=1, \ldots, m-K: D_{k}^{(m-K)} \geq 1\right] P_{m}[D=\infty] .
$$

Applying the strong Markov property once more, this time to $T_{m-K}$, and using the i.i.d. structure of the environment, we get that the above is equal to

$$
\begin{aligned}
& \sum_{m \geq K} P_{m-K}\left[\vec{D}^{(m)}=\vec{i}\right] P_{0}\left[\forall k=1, \ldots, m-K: D_{k}^{(m-K)} \geq 1\right] P_{m}[D=\infty] \\
& =P_{0}\left[\vec{D}^{(K)}=\vec{i}\right] \sum_{m \geq K} P_{0}\left[\forall k=1, \ldots, m-K: D_{k}^{(m-K)} \geq 1\right] P_{m-K}[D=\infty] \\
& \quad=P_{0}\left[\vec{D}^{(K)}=\vec{i}\right] \sum_{m \geq K} P_{0}\left[\tau_{1}=T_{m-K}\right]=P_{0}\left[\vec{D}^{(K)}=\vec{i}\right] .
\end{aligned}
$$

This proves (35). Now we need to show that

$$
P_{0}\left[\vec{D}^{(K)}=\vec{i}\right]=P_{0}[\vec{V}=\vec{i}] .
$$

The proof is essentially the same as that of Proposition 2.2 of [BS08a]. At first, notice that, given $\left(D_{1}^{(K)}, \ldots, D_{k}^{(K)}\right)$, the distribution of $D_{k+1}^{(K)}$ depends only on $D_{k}^{(K)}$. Therefore, the process $\left(D_{k}^{(K)}\right)_{0 \leq k \leq K}$ 
is Markov, just as the process $\left(\widetilde{V}_{k}\right)_{0 \leq k \leq K}$. Both processes get absorbed after the first return to 0 . Then (39) will follow if we show that they have the same transition probabilities and that $D_{1}^{(K)}$ has the same distribution as $\widetilde{V}_{1}$. Let $m \geq 1$ and $1 \leq k \leq K-1$ or $m=k=0$. Notice that if the number $D_{k}^{(K)}$ of downcrossings of the edge $(K-k, K-k-1)$ prior to $T_{K}$ is $m$ then the number of upcrossings of the same edge prior to $T_{K}$ equals $m+1$. Therefore, the number $D_{k+1}^{(K)}$ of downcrossings of the edge $(K-k-1, K-k-2)$ prior to $T_{K}$ is equal to the number of failures in $\left(Y_{i}^{(K-k-1)}\right)_{i \geq 1}$ before the $(m+1)$-st success, which is $F_{m+1}^{(K-k-1)}$. On the other hand, if $\widetilde{V}_{k}=m$ then, by (32) and (33), $\widetilde{V}_{k+1}=F_{m+1}^{(k)}$. But for all $i, j \geq 0$ random variables $F_{m+1}^{(i)}$ and $F_{m+1}^{(j)}$ have the same distribution.

\section{Law of large numbers and ballisticity}

While (25) gives the law of large numbers in the transient case the renewal structure does not say anything about the recurrent case. The following general result covers both the transient and the recurrent case.

Proposition 13. There is a deterministic $v \in[-1,1]$ such that $P_{0}$-a.s. $X_{n} / n \rightarrow v$ for $n \rightarrow \infty$.

Proof. It can be shown exactly like in the proof of [Zer05, Theorem 13] that if $\sup _{n \geq 0} X_{n}=\infty$ a.s. then

$$
\begin{aligned}
\limsup _{n \rightarrow \infty} \frac{X_{n}}{n} & \leq \frac{1}{u_{+}} \quad \text { a.s., where } \\
u_{+} & :=\sum_{j \geq 1} P_{0}\left[T_{j+1}-T_{j} \geq j\right] \in[1, \infty], \quad \text { and } \\
\liminf _{n \rightarrow \infty} \frac{X_{n}}{n} & \geq \frac{1}{u_{+}} \quad \text { a.s. if } u_{+}<\infty
\end{aligned}
$$

see the last line on p. 113 and the first line on p. 114 of [Zer05]. Similarly, by symmetry, if $\inf _{n \geq 0} X_{n}=-\infty$ a.s. then

$$
\begin{aligned}
\liminf _{n \rightarrow \infty} \frac{X_{n}}{n} & \geq \frac{-1}{u_{-}} \quad \text { a.s., where } \\
u_{-} & :=\sum_{j \geq 1} P_{0}\left[T_{-j-1}-T_{-j} \geq j\right] \in[1, \infty], \text { and } \\
\limsup _{n \rightarrow \infty} \frac{X_{n}}{n} & \leq \frac{-1}{u_{-}} \text {a.s. if } u_{-}<\infty .
\end{aligned}
$$

Now due to Theorem 1 there are only three cases: Either the walk is transient to the right or it is transient to the left or it is recurrent. Consider the case of transience to the right. If $u_{+}<\infty$ then $\lim _{n} X_{n} / n=1 / u_{+}$follows directly from (40) and (41). If $u_{+}=\infty$ then $\lim _{n} X_{n} / n=0$ follows from (40) and $\inf _{n} X_{n}>-\infty$. Transience to the left is treated analogously. In the case of recurrence we have a.s. both $\sup _{n} X_{n}=\infty$ and $\inf _{n} X_{n}=-\infty$. Hence both $u_{+}$and $u_{-}$are infinite due to (41) and (43), respectively. Therefore, by (40) and (42), $\lim _{n} X_{n} / n=0$. 
Lemma 14. Let $\left(Z_{k}\right)_{k \geq 0}$ be a (Geom(1/2), $\left.v\right)$-branching process, where $v$ is the distribution of $\eta_{k}:=$ $F_{M}^{(k)}-M+1$, and recall definitions (8), (32) and (33). Then

$$
E_{0}[\widetilde{V}]<\infty \Longleftrightarrow E_{0}[\widetilde{Z}]<\infty \text {, where } \widetilde{V}:=\sum_{k \geq 0} \widetilde{V}_{k} \text { and } \tilde{Z}:=\sum_{k \geq 0} \widetilde{Z}_{k}
$$

Proof. As an intermediate step we first consider the auxiliary Markov chain $\left(W_{k}\right)_{k \geq 0}$ defined by

$$
W_{0}:=0, \quad W_{k+1}:=F_{\left(W_{k}+1\right) \vee M}^{(k)} .
$$

This is a branching process with migration in the following sense: At each step, it exhibits two types of behavior: 1) if $W_{k} \geq M-1$ then one particle immigrates and then all $W_{k}+1$ particles reproduce; 2) if $W_{k}<M-1$ then $M-W_{k}$ particles immigrate and then all $M$ particles reproduce.

We shall first establish the equivalence

$$
E_{0}[\widetilde{V}]<\infty \Longleftrightarrow E_{0}[\widetilde{W}]<\infty
$$

where, as usual,

$$
N^{(W)}:=\inf \left\{k \geq 1 \mid W_{k}=0\right\}, \quad \widetilde{W}_{k}:=W_{k} \mathbf{1}_{\left\{k<N^{(W)}\right\}}, \text { and } \widetilde{W}:=\sum_{k \geq 0} \widetilde{W}_{k} .
$$

Comparing definitions (32) and (45) we see that $\widetilde{V}_{k} \leq \widetilde{W}_{k}$ for all $k$, which yields the implication $\Leftarrow$ in (46). For the reverse implication, assume that $E_{0}[\widetilde{V}]$ is finite. Since $N^{(V)} \leq \widetilde{V}+1$ this implies that $\left(V_{k}\right)_{k \geq 0}$ is positive recurrent. The following lemma, whose proof is postponed, will help us to compare $\left(V_{k}\right)_{k \geq 0}$ and $\left(W_{k}\right)_{k \geq 0}$.

Lemma 15. Let $K$ be the transition matrix of a positive recurrent Markov chain with state space $\mathbb{N}_{0}$ and invariant distribution $\pi$. Assume also that all entries of $K$ are strictly positive. Fix a state $j \in \mathbb{N}_{0}$ and a finite set $J \subset \mathbb{N}_{0} \backslash\{j\}$. Modify a finite number of rows of $K$ by setting

$$
\bar{K}(i, \cdot):= \begin{cases}K(i, \cdot), & \text { if } i \notin J \\ K(j, \cdot), & \text { if } i \in J\end{cases}
$$

Then a Markov chain with the transition matrix $\bar{K}$ is also positive recurrent and its unique invariant probability distribution $\bar{\pi}$ satisfies $\bar{\pi}(n) \leq c_{6} \pi(n)$ for all $n \in \mathbb{N}_{0}$.

If we let $K$ be the transition matrix of the Markov chain $\left(V_{k}\right)_{k \geq 0}$ and set $j=M-1$ and $J=$ $\{0,1, \ldots, M-2\}$ then $\bar{K}$ defined in Lemma 15 is the transition matrix of $\left(W_{k}\right)_{k \geq 0}$. Moreover, all entries of this $K$ are strictly positive due to (2). Consequently, we may apply Lemma 15 and get that $\left(W_{k}\right)_{k \geq 0}$ is positive recurrent and its invariant probability distribution $\bar{\pi}$ is bounded above by a multiple $c_{6} \pi$ of the invariant probability distribution $\pi$ of $\left(V_{k}\right)_{k \geq 0}$. By Theorem 5.4.3 of [Du05], $\pi$ and $\bar{\pi}$ can be represented as $\pi=\rho / E_{0}\left[N^{(V)}\right]$ and $\bar{\pi}=\bar{\rho} / E_{0}\left[N^{(\bar{W})}\right]$, where for $s \in \mathbb{N}_{0}$,

$$
\rho(s):=E_{0}\left[\sum_{k=0}^{N^{(V)}-1} \mathbf{1}_{\left\{V_{k}=s\right\}}\right] \text { and } \bar{\rho}(s):=E_{0}\left[\sum_{k=0}^{N^{(W)}-1} \mathbf{1}_{\left\{W_{k}=s\right\}}\right] \text {. }
$$


Therefore, also $\bar{\rho} \leq c_{7} \rho$. However,

$$
E_{0}[\widetilde{W}]=E_{0}\left[\sum_{k=0}^{N^{(W)}-1} \sum_{s \geq 0} s \mathbf{1}_{\left\{W_{k}=s\right\}}\right]=\sum_{s \geq 0} s \bar{\rho}(s)
$$

and, similarly, $E_{0}[\widetilde{V}]=\sum_{s} s \rho(s)$. Consequently, $E_{0}[\widetilde{W}] \leq c_{7} E_{0}[\widetilde{V}]$. This gives the implication $\Rightarrow$ in (46). Next, we show that

$$
E_{0}[\widetilde{W}]<\infty \Longleftrightarrow E_{0}[\widetilde{Z}]<\infty .
$$

As in Lemma 6 we decompose the process $\left(W_{k}\right)_{k \geq 0}$ into two components.

Lemma 16. For $k \geq 0$ let $Z_{k}^{\prime}:=W_{k+1}-F_{M}^{(k)}$. Then $\left(Z_{k}^{\prime}\right)_{k \geq 0}$ is a (Geom(1/2),v)-branching process, where $v$ is the common distribution of $\eta_{k}:=F_{M}^{(k)}-M+1$ under $P_{1}$.

The proof of Lemma 16 is almost identical to the one of Lemma 6 and, thus, is omitted.

Since $F_{M}^{(k)} \geq 0$, we immediately obtain $\widetilde{Z}_{k}^{\prime} \leq \widetilde{W}_{k+1}$, where $\widetilde{Z}_{k}^{\prime}$ is defined by replacing $W$ in (47) by $Z^{\prime}$. By Lemma $16,\left(\widetilde{Z}_{k}\right)_{k}$ and $\left(\widetilde{Z}_{k}^{\prime}\right)_{k}$ have the same distribution. Therefore $E_{0}[\widetilde{Z}]=E_{0}\left[\widetilde{Z}^{\prime}\right] \leq E_{0}[\widetilde{W}]$, which yields the implication $\Rightarrow$ in (49). For the opposite direction assume that $E_{0}[\widetilde{Z}]<\infty$. Then, as in the proof of (46), $\left(Z_{k}^{\prime}\right)_{k \geq 0}$ is positive recurrent and, by the equivalent of (48), its invariant distribution, say $\pi^{\prime}$, has a finite mean. Since $Z_{k}^{\prime}$ and $F_{M}^{(k)}$ are independent, it follows from Lemma 16 that the convolution of $\pi^{\prime}$ and the distribution of $F_{M}^{(k)}$ is invariant for $\left(W_{k}\right)_{k \geq 0}$. This convolution has a finite mean as well, which implies, as in (48), that $E_{0}[\widetilde{W}]$ is finite as well. This concludes the proof of (49). The statement of the lemma now follows from (46) and (49).

Proof of Lemma 15. It suffices to consider the case in which $J$ has only one element, i.e. $J=\{i\}$ for some $i \neq j$. The full statement then follows by induction, changing one row at a time. Let $\left(\zeta_{k}\right)_{k \geq 0}$ and $\left(\bar{\zeta}_{k}\right)_{k \geq 0}$ be Markov chains with transition matrices $K$ and $\bar{K}$, respectively. Their initial point will be denoted by a subscript of $P$ and $E$. Since all the entries of $\bar{K}$ are strictly positive, $\bar{K}$ is irreducible. It is recurrent, since its state $i$ is recurrent. Indeed, $P_{i}\left[\exists k \geq 1: \bar{\zeta}_{k}=i\right]=P_{j}\left[\exists k \geq 1: \bar{\zeta}_{k}=i\right]$ because of $\bar{K}(i, \cdot)=K(j, \cdot)=\bar{K}(j, \cdot)$. Moreover, since $\left(\zeta_{k}\right)_{k \geq 0}$ and $\left(\bar{\zeta}_{k}\right)_{k \geq 0}$ are indistinguishable as long as they do not touch $i$, i.e. since $K(s, \cdot)=\bar{K}(s, \cdot)$ for all $s \neq i$, we can switch from the process $\left(\bar{\zeta}_{k}\right)_{k \geq 0}$ to $\left(\zeta_{k}\right)_{k \geq 0}$ and obtain that $P_{i}\left[\exists k \geq 1: \bar{\zeta}_{k}=i\right]=P_{j}\left[\exists k \geq 1: \zeta_{k}=i\right]$, which is equal to 1 because $\left(\zeta_{k}\right)_{k \geq 0}$ is recurrent.

Similarly, one can show that $\left(\bar{\zeta}_{k}\right)_{k \geq 0}$ is also positive recurrent. Define the hitting time $\sigma:=\inf \{k \geq$ $\left.1 \mid \zeta_{k}=i\right\}$ for $\left(\zeta_{k}\right)_{k \geq 0}$ and analogously $\bar{\sigma}$ for $\left(\bar{\zeta}_{k}\right)_{k \geq 0}$. Then, again by [Du05, Theorem 5.4.3], $\rho$ and $\bar{\rho}$, defined by

$$
\rho(s):=E_{i}\left[\sum_{k=0}^{\sigma-1} \mathbf{1}_{\left\{\zeta_{k}=s\right\}}\right] \quad \text { and } \bar{\rho}(s):=E_{i}\left[\sum_{k=0}^{\bar{\sigma}-1} \mathbf{1}_{\left\{\bar{\zeta}_{k}=s\right\}}\right], \quad s \in \mathbb{N}_{0},
$$

are invariant measures for $K$ and $\bar{K}$, respectively. Using the relations between $K$ and $\bar{K}$ as above, we have for all $s \in \mathbb{N}_{0}$,

$$
\bar{\rho}(s)=E_{j}\left[\sum_{k=0}^{\bar{\sigma}-1} \mathbf{1}_{\left\{\bar{\zeta}_{k}=s\right\}}\right]=E_{j}\left[\sum_{k=0}^{\sigma-1} \mathbf{1}_{\left\{\zeta_{k}=s\right\}}\right] .
$$


On the other hand, for all $s \in \mathbb{N}_{0}$,

$$
\rho(s) \geq E_{i}\left[\sum_{k=1}^{\sigma-1} \mathbf{1}_{\left\{\zeta_{1}=j, \zeta_{k}=s\right\}}\right]=K(i, j) E_{j}\left[\sum_{k=0}^{\sigma-1} \mathbf{1}_{\left\{\zeta_{k}=s\right\}}\right] \stackrel{(50)}{=} K(i, j) \bar{\rho}(s) .
$$

Since $\left(\zeta_{k}\right)_{k \geq 0}$ is positive recurrent, $\rho$ 's total mass, $E_{i}[\sigma]$, is finite. Consequently, by the above and since $K(i, j)>0$, $\bar{\rho}$ 's total mass, $E_{i}[\bar{\sigma}]$, is finite as well. Therefore, $\left(\bar{\zeta}_{k}\right)_{k \geq 0}$ is positive recurrent and its invariant measure $\bar{\pi}$ satisfies $\bar{\pi} \leq c_{6} \pi$ with $c_{6}:=E_{i}[\sigma] /\left(E_{i}[\bar{\sigma}] K(i, j)\right)$.

The following lemma is the counterpart of Lemma 10.

Lemma 17. Let $v$ be the distribution of $F_{M}^{(0)}-M+1$ under $P_{0}$. Then $\theta$ defined in (7) for the $(\operatorname{Geom}(1 / 2), v)$-branching process is equal to $1-\delta$.

Proof. As in the proof of Lemma $10, \theta=E_{0}\left[F_{M}^{(0)}\right]-M+1$ since $b=1$. Switching failures and successes in (23) and replacing $\omega(x, i)$ by $1-\omega(x, i)$ yields $E_{0}\left[F_{M}^{(0)}\right]-M=-\delta$.

Proof of Theorem 2. The first statement of the theorem, the existence of the velocity $v$, is just Proposition 13, or $(25)$ in the transient case. If $\delta \in[-1,1]$ then the walk is recurrent by Theorem 1 and therefore $v=0$.

Now let $|\delta|>1$. Without loss of generality we may assume $\delta>1$. Then the walk is transient to the right by Theorem 1. By (26), $v>0$ iff $E_{0}\left[\tau_{2}-\tau_{1}\right]<\infty$. By Lemma 11 with $p=1$ this is the case iff $E_{0}\left[\sum_{k>0} D_{k}\right]<\infty$. By Lemma 12 this holds iff $E_{0}\left[\sum_{k \geq 0} \widetilde{V}_{k}\right]<\infty$. Due to Lemma 14 this is true iff $E_{0}\left[\sum_{k \geq 0} \widetilde{Z}_{k}\right]$ is finite. By the second statement in Corollary 4 this holds iff $\theta<-1$. Thus, by Lemma $17, v>0$ iff $\delta>2$.

\section{Central limit theorem}

Lemma 18. Let $\left(\widetilde{V}_{k}\right)_{k \geq 0}$ be defined by (33). Then $\delta>4$ implies that the random variable $\widetilde{V}:=\sum_{k \geq 0} \widetilde{V}_{k}$ has a finite second moment.

Proof. Let $\left(W_{k}\right)_{k \geq 0}$ and $\widetilde{W}$ be defined by (45) and (47), respectively, using the same sequences $\left(F_{i}^{(k)}\right)_{i \geq 0}, k \in \mathbb{N}_{0}$, as for the process $\left(V_{k}\right)_{k \geq 0}$. Since $V_{k} \leq W_{k}$, we have $N^{(V)} \leq N^{(W)}$ and $E_{0}\left[\widetilde{V}^{2}\right] \leq$ $E_{0}\left[\widetilde{W}^{2}\right]$. We shall prove that the latter is finite. By Minkowski's inequality we have

$$
\left(E_{0}\left[\widetilde{W}^{2}\right]\right)^{1 / 2}=\left(E_{0}\left[\left(\sum_{k \geq 0} W_{k} \mathbf{1}_{\left\{N^{(W)}>k\right\}}\right)^{2}\right]\right)^{1 / 2} \leq \sum_{k \geq 0}\left(E_{0}\left[W_{k}^{2} \mathbf{1}_{\left\{N^{(W)}>k\right\}}\right]\right)^{1 / 2} .
$$

From Lemma 16 we see that $Z_{k}:=W_{k+1}-F_{M}^{(k)}$ defines a (Geom(1/2), $\left.v\right)$-branching process, where $v$ is the distribution of $F_{M}^{(0)}-M+1$. Therefore, $W_{k}^{2} \leq 2\left(Z_{k-1}^{2}+\left(F_{M}^{(k-1)}\right)^{2}\right)$. Combining this with the fact that $(a+b)^{1 / 2} \leq a^{1 / 2}+b^{1 / 2}$ for all $a, b \geq 0$, we get

$$
\begin{aligned}
\left(E_{0}\left[\widetilde{W}^{2}\right]\right)^{1 / 2} & \leq \sqrt{2} \sum_{k=1}^{\infty}\left(E_{0}\left[Z_{k-1}^{2} \mathbf{1}_{\left\{N^{(W)}>k\right\}}\right]+E_{0}\left[\left(F_{M}^{(k-1)}\right)^{2} \mathbf{1}_{\left\{N^{(W)}>k\right\}}\right]\right)^{1 / 2} \\
& \leq \sqrt{2} \sum_{k=1}^{\infty}\left[\left(E_{0}\left[Z_{k-1}^{2} \mathbf{1}_{\left\{N^{(W)}>k\right\}}\right]\right)^{1 / 2}+\left(E_{0}\left[\left(F_{M}^{(k-1)}\right)^{2} \mathbf{1}_{\left\{N^{(W)}>k\right\}}\right]\right)^{1 / 2}\right] .
\end{aligned}
$$


Applying Hölder's inequality with $1 / \alpha+1 / \alpha^{\prime}=1, \alpha>1$, we obtain

$$
\begin{aligned}
\left(E_{0}\left[\widetilde{W}^{2}\right]\right)^{1 / 2} \leq \sqrt{2} \sum_{k=1}^{\infty}\left(E_{0}\left[Z_{k-1}^{2 \alpha}\right]\right)^{1 /(2 \alpha)} & \left(P_{0}\left[N^{(W)}>k\right]\right)^{1 /\left(2 \alpha^{\prime}\right)} \\
& +\sqrt{2} E_{0}\left[\left(F_{M}^{(0)}\right)^{2 \alpha}\right]^{1 /(2 \alpha)} \sum_{k=1}^{\infty}\left(P_{0}\left[N^{(W)}>k\right]\right)^{1 /\left(2 \alpha^{\prime}\right)} .
\end{aligned}
$$

We are going to show that

(i) for every $\varepsilon \in(0, \delta-4)$ there is a constant $c_{8}(\varepsilon, \delta)$ such that

$$
P_{0}\left[N^{(W)}>k\right] \leq c_{8} k^{-\delta+\varepsilon} \quad \text { for all } k \in \mathbb{N} \text {; }
$$

(ii) for each $\ell \in \mathbb{N}$ there is a constant $c_{9}(\ell)$ such that $E_{0}\left[Z_{k}^{\ell}\right] \leq c_{9}(\ell) k^{\ell}$ for all $k \in \mathbb{N}_{0}$.

Let us assume (i) and (ii) for the moment and see that both series in the right hand side of (51) are finite. Choose $\alpha^{\prime} \in(1, \delta / 4)$ so that $\alpha=\alpha^{\prime} /\left(\alpha^{\prime}-1\right)$ is an integer and let $\varepsilon=\left(\delta-4 \alpha^{\prime}\right) / 2$. Then by (i) and (ii) for all $k \geq 1$,

$$
\left(E_{0}\left[Z_{k-1}^{2 \alpha}\right]\right)^{1 /(2 \alpha)}\left(P_{0}\left[N^{(W)}>k\right]\right)^{1 /\left(2 \alpha^{\prime}\right)} \leq c_{10}\left(\alpha^{\prime}, \delta\right) k^{1-(\delta-\varepsilon) /\left(2 \alpha^{\prime}\right)}=c_{10} k^{-\delta /\left(4 \alpha^{\prime}\right)} .
$$

Since $\delta /\left(4 \alpha^{\prime}\right)>1$, the first series in the right hand side of (51) converges. It is obvious now that for the same choice of $\alpha^{\prime}$ and $\varepsilon$ the second series in the right hand side of (51) also converges. Therefore we only need to prove (i) and (ii).

Proof of (i). Observe that $W_{k}$ is zero if and only if both $Z_{k-1}$ and $F_{M}^{(k-1)}$ are equal to zero. Set $N_{0}:=0$ and consider the times $N_{i}:=\inf \left\{k>N_{i-1} \mid Z_{k}=0\right\}, i \in \mathbb{N}$, when the process $\left(Z_{k}\right)_{k \geq 1}$ dies out. Due to Lemma 17 and $\delta>4$ the parameter $\theta$ for the process $\left(Z_{k}\right)_{k \geq 0}$ satisfies

$$
\theta=1-\delta<-3 \text {. }
$$

In particular, Corollary 4 implies that the process $\left(Z_{k}\right)_{k \geq 0}$ is positive recurrent. Therefore, all $N_{i}, i \in$ $\mathbb{N}$, are a.s. finite and $\left(N_{i}-N_{i-1}\right)_{i \in \mathbb{N}}$ is i.i.d.. We are interested in the sequence $\left(F_{M}^{\left(N_{i}\right)}\right)_{i \in \mathbb{N}}$. Observe that $\left(F_{M}^{(n)}\right)_{n \geq 0}$ is i.i.d. and, by construction, $F_{M}^{(n)}$ is independent from $\mathscr{F}_{n}=\sigma\left(\left\{Z_{k}, k \leq n\right\},\left\{F_{M}^{(j)}, j<n\right\}\right)$. It is then straightforward to check that each $F_{M}^{\left(N_{i}\right)}$ is independent from $\mathscr{F}_{N_{i}}, i \in \mathbb{N}$, and $\left(F_{M}^{\left(N_{i}\right)}\right)_{i \geq 1}$ is i.i.d.. Let $\chi:=\inf \left\{i \geq 1 \mid F_{M}^{\left(N_{i}\right)}=0\right\}$. Then $\chi$ has the geometric distribution on $\mathbb{N}$ with parameter $p:=P\left[F_{M}^{(0)}=0\right] \in(0,1)$. Therefore, $N^{(W)}=N_{\chi}=\sum_{i=1}^{\chi}\left(N_{i}-N_{i-1}\right)$ and

$$
\begin{aligned}
P_{0}\left[N^{(W)}>k\right] & =P_{0}\left[N_{\chi}^{\delta-\varepsilon}>k^{\delta-\varepsilon}\right] \leq \frac{1}{k^{\delta-\varepsilon}} \sum_{m \geq 1} E_{0}\left[N_{m}^{\delta-\varepsilon} \mathbf{1}_{\{\chi=m\}}\right] \\
& \leq \frac{1}{k^{\delta-\varepsilon}} \sum_{m \geq 1}\left(E_{0}\left[N_{m}^{(\delta-\varepsilon) \beta}\right]\right)^{1 / \beta}\left(P_{0}[x=m]\right)^{1 / \beta^{\prime}}
\end{aligned}
$$

where $\beta \in(1, \delta /(\delta-\varepsilon)), \beta^{\prime}=\beta /(\beta-1)$. Denote $(\delta-\varepsilon) \beta$ by $\gamma$. Writing $N_{m}$ as $\sum_{i=1}^{m}\left(N_{i}-N_{i-1}\right)$ and using Minkowski's inequality we get $\left(E_{0}\left[N_{m}^{\gamma}\right]\right)^{1 / \gamma} \leq m\left(E_{0}\left[N_{1}^{\gamma}\right]\right)^{1 / \gamma}$. Substituting this into (53) we obtain

$$
P_{0}\left[N^{(W)}>k\right] \leq \frac{\left(E_{0}\left[N_{1}^{\gamma}\right]\right)^{1 / \beta}}{k^{\delta-\varepsilon}} \sum_{m=1}^{\infty} m^{\delta-\varepsilon}\left((1-p)^{m-1} p\right)^{1 / \beta^{\prime}} \leq \frac{\left(E_{0}\left[N_{1}^{\gamma}\right]\right)^{1 / \beta}}{k^{\delta-\varepsilon}} c_{11}(\varepsilon, \delta) .
$$


From part (iv) of Theorem A and (52) we know that $P_{0}\left[N_{1}>k\right] \sim c_{4} k^{-\delta}$. Therefore, and since $\gamma<\delta$ by assumption, $P_{0}\left[N_{1}^{\gamma}>k\right]$ is summable in $k$. Consequently, $E_{0}\left[N_{1}^{\gamma}\right]=c_{12}(\varepsilon, \delta)<\infty$. This implies (i).

Proof of (ii). The proof can be easily done by induction in $\ell$. The statement is trivial for $\ell=0$. (Here $0^{0}=1$.) Assume now $\ell \geq 1$ and that for each $j \in\{0,1,2, \ldots, \ell-1\}$ there is a constant $c_{9}(j)$ such that $E_{0}\left[Z_{k}^{j}\right] \leq c_{9}(j) k^{j}$ for all $k \in \mathbb{N}_{0}$. Using that $\left(Z_{k}\right)_{k}$ is a $(\operatorname{Geom}(1 / 2), v)$-branching process we have for all $k \in \mathbb{N}_{0}$ and $n \in \mathbb{N}$,

$$
\begin{aligned}
E_{0}\left[Z_{k+1}^{\ell}\right]= & E_{0}\left[\left(\xi_{1}^{(k+1)}+\xi_{2}^{(k+1)}+\cdots+\xi_{Z_{k}+\eta_{k}}^{(k+1)}\right)^{\ell}\right] \\
= & E_{0}\left[\left(\xi_{1}^{(k+1)}+\cdots+\xi_{Z_{k}+\eta_{k}}^{(k+1)}\right)^{\ell} 1_{\left\{Z_{k}+\eta_{k} \leq \ell\right\}}\right] \\
& \quad+\sum_{n>\ell} E_{0}\left[\left(\xi_{1}^{(k+1)}+\cdots+\xi_{Z_{k}+\eta_{k}}^{(k+1)}\right)^{\ell} \mathbf{1}_{\left\{Z_{k}+\eta_{k}=n\right\}}\right] \\
\leq E_{0}\left[\left(\xi_{1}^{(0)}+\cdots+\xi_{\ell}^{(0)}\right)^{\ell}\right] & \quad+\sum_{n>\ell} E_{0}\left[\left(\xi_{1}^{(0)}+\cdots+\xi_{n}^{(0)}\right)^{\ell}\right] P_{0}\left[Z_{k}+\eta_{k}=n\right]
\end{aligned}
$$

Observe that the expectation in (54) is bounded by a constant $c_{13}(\ell)$. To control the series in (55) we use the following lemma, whose proof is postponed until after the end of the present proof.

Lemma 19. Let $\left(\xi_{i}\right)_{i \in \mathbb{N}}$ be non-negative i.i.d. random variables such that $E\left[\xi_{1}\right]=1$ and $E\left[\xi_{1}^{\ell}\right]<\infty$ for some positive integer $\ell$. Then there is a constant $c_{14}$ such that for all $n>\ell$,

$$
E\left[\left(\xi_{1}+\xi_{2}+\cdots+\xi_{n}\right)^{\ell}\right] \leq n^{\ell}+c_{14} \sum_{m=1}^{\ell-1} n^{m}
$$

Applying this lemma to the series in (55) we obtain

$$
\begin{aligned}
E_{0}\left[Z_{k+1}^{\ell}\right] & \leq c_{13}+\sum_{n>\ell}\left(n^{\ell}+c_{14} \sum_{m=1}^{\ell-1} n^{m}\right) P_{0}\left[Z_{k}+\eta_{k}=n\right] \\
& \leq c_{13}+E_{0}\left[\left(Z_{k}+\eta_{k}\right)^{\ell}\right]+c_{14} \sum_{m=1}^{\ell-1} E_{0}\left[\left(Z_{k}+\eta_{k}\right)^{m}\right] \\
& \leq E_{0}\left[Z_{k}^{\ell}\right]+\sum_{m=0}^{\ell-1} c_{15}(m, \ell) E_{0}\left[Z_{k}^{m}\right]
\end{aligned}
$$

By the induction hypothesis we conclude that $E_{0}\left[Z_{k+1}^{\ell}-Z_{k}^{\ell}\right] \leq c_{16} k^{\ell-1}$. Summation over $k$ implies (ii) and finishes the proof of Lemma 18.

Proof of Lemma 19. By expanding and using independence,

$$
E\left[\left(\xi_{1}+\xi_{2}+\cdots+\xi_{n}\right)^{\ell}\right]=\sum_{m=1}^{\ell}\left(\begin{array}{c}
n \\
m
\end{array}\right) \sum_{\substack{\ell_{1}+\ldots+\ell_{m}=\ell \\
1 \leq \ell_{1}, \ldots, \ell_{m}}}\left(\begin{array}{c}
\ell \\
\ell_{1}, \ldots, \ell_{m}
\end{array}\right) \prod_{j=1}^{m} E\left[\xi_{1}^{\ell_{j}}\right] .
$$


Since $E\left[\xi_{1}\right]=1$ the summand for $m=\ell$ is equal to $n ! /(n-\ell) ! \leq n^{\ell}$. For the other terms we estimate the factor $\left(\begin{array}{l}n \\ m\end{array}\right)$ from above by $n^{m}$ and define the constant

$$
c_{14}:=\max _{1 \leq m<\ell} \sum_{\substack{\ell_{1}+\cdots+\ell_{m}=\ell \\
1 \leq \ell_{1}, \ldots, \ell_{m}}}\left(\begin{array}{c}
\ell \\
\ell_{1}, \ldots, \ell_{m}
\end{array}\right) \prod_{j=1}^{m} E\left[\xi_{1}^{\ell_{j}}\right] .
$$

Proof of Theorem 3. Let $|\delta|>4$. By symmetry we may assume without loss of generality that $\delta>4$. Then, by Lemma $18, E_{0}\left[\left(\sum_{k \geq 1} \widetilde{V}_{k}\right)^{2}\right]<\infty$. By Lemma 12 this implies $E_{0}\left[\left(\sum_{k} D_{k}\right)^{2}\right]<\infty$. Lemma 11 for $p=2$ gives $E_{0}\left[\left(\tau_{2}-\tau_{1}\right)^{2}\right]<\infty$. This implies the claim, as indicated in (27) and (28).

\section{Further remarks, a multi-dimensional example, and open questions}

Remark 3 (Permuting cookies). Note that permuting cookies within the cookie piles, i.e. replacing $(\omega(\cdot, i))_{i \geq 1}$ by $\omega(\cdot, \pi(i))_{i \geq 1}$, where $\pi: \mathbb{N} \rightarrow \mathbb{N}$ is a permutation, does not change $\delta$, defined in (3). Therefore, permuting cookies does not change the classification of the walk as described in Theorems 1 and 2. This fact can also be seen as follows without using the results in Theorem A from the literature.

At first, observe that we may assume without loss of generality that $\pi$ is a finite permutation, since all cookies in a cookie pile except for a finite number are placebo cookies. We may even assume that all $i>M$ are fixed points for $\pi$. Indeed, otherwise just replace the original $M$ with $\max \{\pi(i) \mid i \leq M\}$. To argue, for example, that permuting cookies does not turn a walk which is recurrent from the right into one which is not recurrent from the right or vice versa, recall the definition of $S_{M}^{(k)}$ in (12) and of $\eta_{k}$ in Lemma 7 and denote by $\sigma_{M}^{(k)} \geq M$ the index of the $M$-th failure in the sequence $\left(Y_{i}^{(k)}\right)_{i \geq 1}$. Then $\eta_{k}$ can be written as

$$
\eta_{k}=\sum_{i=1}^{\sigma_{M}^{(k)}} Y_{i}^{(k)}=\sum_{i=1}^{M} Y_{i}^{(k)}+\sum_{i=M+1}^{\sigma_{M}^{(k)}} Y_{i}^{(k)}=\sum_{i=1}^{M} Y_{\pi(i)}^{(k)}+\sum_{i=M+1}^{\sigma_{M}^{(k)}} Y_{\pi(i)}^{(k)},
$$

where we used in the last step for the first sum that $\pi$ maps $\{1, \ldots, M\}$ onto itself and for the second sum that all $i>M$ are fixed points for $\pi$. For the same reason, $\sigma_{M}^{(k)} \geq M$ is also the index of the $M$-th failure in the permuted sequence $Y_{\pi(i)}^{(k)}$. This shows that applying $\pi$ does not change the distribution $v$ of $\eta_{k}$. Therefore, recurrence to the right, as characterized in Lemma 7, is invariant under permutations. Since the proof of Lemma 7 did not use any results from Section 2, this proof is self-contained.

Similarly, one can show that the positivity of speed is invariant under permutations. Indeed, in the proof of Theorem 2 we have shown that $v>0$ iff $E_{0}\left[\sum_{k \geq 0} \widetilde{Z}_{k}\right]<\infty$. By the above argument, the distribution of $\left(\widetilde{Z}_{k}\right)_{k \geq 0}$ remains unchanged under permutations.

Remark 4 (Higher dimensions). Multi-dimensional ERW with cookies that induce a bias with a nonnegative projection in some fixed direction were considered in [BW03], [Ko03], [Ko05], [Zer06], [HH06] and [BR07]. A special non-stationary cookie environment with two types of cookies pointing into opposite directions was studied in [ABK08]. However, to the best of our knowledge so far 
there are no criteria for recurrence, transience or ballistic behavior of ERWs in i.i.d. environments of "positive" and "negative" cookies in higher dimensions. To us, it is not even clear how such criteria could look like. In the following example we shall indicate that the situation cannot be as simple as in one dimension. We shall show that, unlike for $d=1$ (see Remark 3), permuting cookies in higher dimensions may change the sign of the velocity.

Example. Let $d \geq 4$. Denote by $\omega(x, e, i)$ the probability for the ERW to jump from $x \in \mathbb{Z}^{d}$ to the nearest neighbor $x+e$ upon the $i$-th visit of $x$. Fix $0<\varepsilon<1 /(2 d)$ and consider the two deterministic cookie environments $\omega_{k}, k=1,2$, defined by

$$
\omega_{k}\left(x, \pm e_{j}, i\right):=\frac{1}{2 d} \pm \varepsilon(-1)^{i+k} \mathbf{1}_{\{j=1 ; i \in\{1,2\}\}}, \quad x \in \mathbb{Z}^{d}, 1 \leq j \leq d, i \geq 1,
$$

where $\left\{e_{1}, \ldots, e_{d}\right\}$ is the canonical basis of $\mathbb{Z}^{d}$. In both environments, $\omega_{1}$ and $\omega_{2}$, there are $M=2$ cookies per site. In the environment $\omega_{1}$ the walk experiences a drift into direction $e_{1}$ upon the first visit to a site and an equal drift into the opposite direction $-e_{1}$ upon the second visit. Otherwise, it behaves just like a simple symmetric random walk. We shall show:

$$
\text { There is some } v>0 \text { such that } \lim _{n \rightarrow \infty} \frac{X_{n}}{n}=v e_{1} \quad P_{0, \omega_{1}} \text {-a.s.. }
$$

The environment $\omega_{2}$ is obtained from $\omega_{1}$ by permuting the two cookies at each site. By symmetry, we obtain from (57) that for the same $v>0$, we have $P_{0, \omega_{2}}$-a.s. $\lim _{n} X_{n} / n=-v e_{1}$. Thus, in this example, permuting two cookies reverses the direction of the speed.

For the proof of (57) denote by $R_{n, 1}:=\left\{X_{m} \mid m<n\right\}$ the range of the walk before time $n \in \mathbb{N}$ and by $R_{n, 2}:=\left\{x \in \mathbb{Z}^{d} \mid \exists k<m<n X_{k}=x=X_{m}\right\}$ the set of vertices which have been visited at least twice before time $n$. It is easy to see that both $\# R_{n, 1}$ and $\# R_{n, 2}$ tend to $\infty$ as $n \rightarrow \infty$. Coupling $\left(X_{n}\right)_{n \geq 0}$ in the natural way to a simple symmetric random walk $\left(S_{n}\right)_{n \geq 0}$ on $\mathbb{Z}^{d}$ yields that $\left(X_{n}\right)_{n \geq 0}$ can be represented as

$$
X_{n}=S_{n}+2 e_{1} a_{n}-2 e_{1} b_{n}, \quad \text { where } \quad a_{n}=\sum_{i=1}^{\# R_{n, 1}} Y_{i, 1} \quad \text { and } \quad b_{n}=\sum_{i=1}^{\# R_{n, 2}} Y_{i, 2}
$$

and $Y_{i, j}(i \geq 1, j=1,2)$ are independent and Bernoulli distributed with parameter $\varepsilon$. Consequently,

$$
\begin{aligned}
\frac{X_{n} \cdot e_{1}}{n} & =\frac{S_{n} \cdot e_{1}}{n}+\frac{2 a_{n}}{\# R_{n, 1}} \frac{\# R_{n, 1}}{n}-\frac{2 b_{n}}{\# R_{n, 2}} \frac{\# R_{n, 2}}{n} \\
& =\frac{S_{n} \cdot e_{1}}{n}+\frac{2 a_{n}}{\# R_{n, 1}} \frac{\# R_{n, 1}-\# R_{n, 2}}{n}+\left(\frac{2 a_{n}}{\# R_{n, 1}}-\frac{2 b_{n}}{\# R_{n, 2}}\right) \frac{\# R_{n, 2}}{n} .
\end{aligned}
$$

The first term on the right hand side of (58) tends to zero $P_{0, \omega_{1}}$-a.s.. The same holds for the last term in (58) since by the strong law of large numbers both $a_{n} / \# R_{n, 1}$ and $b_{n} / \# R_{n, 2}$ tend to $\varepsilon$ as $n \rightarrow \infty$ and $\# R_{n, 2} / n$ is bounded. Therefore,

$$
\liminf _{n \rightarrow \infty} \frac{X_{n} \cdot e_{1}}{n} \geq 2 \varepsilon \liminf _{n \rightarrow \infty} \frac{\# R_{n, 1}-\# R_{n, 2}}{n}
$$

To bound the right hand side of (59) from below we introduce the projection $\pi: \mathbb{Z}^{d} \rightarrow \mathbb{Z}^{d-1}$ defined by $\pi\left(x_{1}, x_{2}, \ldots, x_{d}\right):=\left(x_{2}, \ldots, x_{d}\right)$ onto the subspace spanned by $e_{2}, \ldots, e_{d}$ and consider 
the process $\left(X_{n}^{\prime}\right)_{n \geq 0}$ defined by $X_{n}^{\prime}:=\pi\left(X_{n}\right)$. Under $P_{0, \omega_{1}},\left(X_{n}^{\prime}\right)_{n \geq 0}$ is a simple symmetric random walk on $\mathbb{Z}^{d-1}$ with holding times which are i.i.d. and geometrically distributed with parameter $1-1 / d$. As above, denote by $R_{n, 1}^{\prime}:=\left\{X_{m}^{\prime} \mid m<n\right\}$ the range of this walk before time $n \in \mathbb{N}$ and by $R_{n, 2}^{\prime}:=\left\{x \in \mathbb{Z}^{d-1} \mid \exists k<m<n X_{k}^{\prime}=x=X_{m}^{\prime}\right\}$ the set of vertices which have been visited at least twice before time $n$ by $\left(X_{n}^{\prime}\right)_{n}$.

Returning to the right hand side of (59) we first note that

$$
\# R_{n, 1}-\# R_{n, 2}=\#\left(R_{n, 1} \backslash R_{n, 2}\right)
$$

is the number of vertices which have been visited exactly once by $\left(X_{n}\right)_{n}$ before time $n$. This number is greater than or equal to the number $\#\left(R_{n, 1}^{\prime} \backslash R_{n, 2}^{\prime}\right)$ of vertices which have been visited exactly once by the projected walk $\left(X_{n}^{\prime}\right)_{n}$ before time $n$. According to [Pi74] this last number satisfies a strong law of large numbers and grows like $p^{2} n$, where $p$ is the probability that $\left(X_{n}^{\prime}\right)_{n}$ never returns to its starting point. Since $d \geq 4$ and simple symmetric random walk in three or more dimensions is transient, we have $p>0$. Therefore, by (59),

$$
\liminf _{n \rightarrow \infty} \frac{X_{n} \cdot e_{1}}{n} \geq 2 \varepsilon p^{2}>0 .
$$

In particular, $\left(X_{n} \cdot e_{1}\right)_{n}$ is transient to the right. Using a renewal structure like in [SZ99] for RWRE and in [BR07] for once-ERW and as outlined in Section 6 this implies that $\left(X_{n} \cdot e_{1}\right)_{n}$ satisfies a strong law of large numbers under $P_{0, \omega_{1}}$, i.e. $P_{0, \omega_{1}}$-a.s., $\left(X_{n} \cdot e_{1}\right) / n \rightarrow v$ for some $v \geq 0$. Due to (60) we even have $v>0$. Since obviously $P_{0, \omega_{1}}$-a.s. $X_{n}^{\prime} / n \rightarrow 0$, this yields the statement (57).

Open questions. We have already mentioned that not much is known about positively and negatively ERW in $d \geq 2$. Below we shall discuss $d=1$. It might be also interesting to consider ERW on strips $\mathbb{Z} \times\{0,1, \ldots, L\}, L \geq 1$.

(a) Recurrent regime. For the case of non-negative cookies and $\delta<1$, D. Dolgopyat has shown, [Do08], (the case of strips was also considered) that, under some assumptions, $\frac{X_{[n t]}}{\sqrt{n}}$ converges in law to the unique pathwise solution $W(t)$ (see [CD99]) of the equation

$$
W(t)=B(t)+\delta\left(\max _{[0, t]} W(t)-\min _{[0, t]} W(t)\right),
$$

where $B(t)$ is the standard Brownian motion. Can this result be extended to positively and negatively excited random walks? What happens in the case $|\delta|=1$ ?

(b) Transient regime with zero linear speed. A.-L. Basdevant and A. Singh obtained in [BS08b] for non-negative (deterministic) cookie environments and $1<\delta \leq 2$ the following results.

1. If $\delta \in(1,2)$ then $\frac{X_{n}}{n^{\delta / 2}}$ converges in law to a random variable $S^{-\delta / 2}$, where $S$ is a positive strictly stable random variable with index $\delta / 2$, i.e. with Laplace transform $E\left[e^{-\lambda S}\right]=e^{-c \lambda^{\delta / 2}}$ for some $c>0$.

2. If $\delta=2$ then $\frac{X_{n}}{n / \log n}$ converges in probability to a positive constant.

Their proof is based on the study of branching processes with migration but uses the assumption that all cookies are non-negative. The same result with essentially the same proof might hold in 
the more general setting studied in the current paper. Is there a result for $\delta=2$ similar to (ii) of [KKS75]?

(c) Transient regime with positive linear speed. We do not know whether our condition $\delta>4$ for the validity of the central limit theorem is optimal. How does the process scale for $\delta \in(2,4]$ ? Is the behavior similar to (iii) and (iv) of [KKS75]?

\section{References}

[ABK08] G. Amir, I. Benjamini and G. Kozma (2008). Excited random walk against a wall. Probab. Theory Related Fields 140, 83 - 102

[AR05] T. Antal and S. REDnER (2005). The excited random walk in one dimension. Journal of Physics. A., 38, no. 12, $2555-2577$

[AN72] K.B. AthreyA and P.E. Ney (1972). Branching processes. Reprint of the 1972 original. Mineola, NY: Dover Publications. xi, 287 p., 2004.

[BS08a] A.-L. Basdevant and A. Singh (2008). On the speed of a cookie random walk. Probab. Theory Related Fields 141, no. 3-4, 625 - 645.

[BS08b] A.-L. BASDEVAnt and A. Singh (2008). Rate of growth of a transient cookie random walk. Electron. J. Probab. 13, no. 26, $811-851$.

[BW03] I. Benjamini and D.B. Wilson (2003). Excited random walk. Elect. Comm. Probab. 8, 86 $-92$

[BR07] J. BÉRARD and A. RAmírez (2007). Central limit theorem for the excited random walk in dimension $d \geq 2$. Elect. Comm. in Probab. 12, no. 30, $303-314$

[CD99] L. Chaumont and R.A. Doney (1999). Pathwise uniqueness for perturbed versions of Brownian motion and reflected Brownian motion. Probab. Theory Related Fields 113, no. $4,519-534$.

[Do08] D. Dolgopyat (2008). Central limit theorem for excited random walk in the recurrent regime. 5 p.. Personal communication.

[Du05] R. DuRrett (2005). Probability: Theory and Examples. Duxbury Press, 3rd edition.

[FY89] Sh.K. Formanov and Makhmud Takha Yasin (1989). Limit theorems for life periods for critical Galton-Watson branching processes with migration. (Russian) Izv. Akad. Nauk UzSSR, Ser. Fiz.-Mat. Nauk, No.1, 40-44

[FYK90] Sh.K. Formanov, Makhmud Takha Yasin and S.V. Kaverin (1990). Life spans of GaltonWatson processes with migration. (Russian) In Asymptotic problems in probability theory and mathematical statistics, edited by T.A. Azlarov and Sh.K. Formanov. Tashkent: Fan. 176 p. (pp. 117-135)

[HH06] R. van DeR HofstAd and M. Holmes (2006). An expansion for self-interacting random walks. Preprint 
[Ke77] H. Kesten. A renewal theorem for random walk in a random environment (1977). Probability (Proc. Sympos. Pure Math. Vol. XXXI, Univ. Illinois, Urbana, Ill., 1976), pp. 67-77. Amer. Math. Soc., Providence, R.I.

[KKS75] H. Kesten, M.V. Kozlov and F. Spitzer (1975). A limit law for random walk in random environment. Compositio Math. 30, 145-168

[Ko03] G. Kozma (2003). Excited random walk in three dimensions has positive speed. Preprint. arXiv:math/0310305v1 [math.PR]

[Ko05] G. Kozma (2005). Excited random walk in two dimensions has linear speed. Preprint. arXiv:math/0512535v1 [math.PR]

[MPV06] Th. Mountford, L.P.R. Pimentel and G. Valle (2006). On the speed of the onedimensional excited random walk in the transient regime. Alea 2, 279-296

[Pi74] J. H. Piтt (1974). Multiple points of transient random walks. Proc. Amer. Math. Soc. 43 no.1, 195-199

[So75] F. Solomon (1975). Random walks in a random environment. Ann. Probab. 3, No. 1, $1-31$

[Sz00] A.-S. Sznitman (2000). Slowdown estimates and central limit theorem for random walks in random environment. J. Eur. Math. Soc. 2, no. 2, 93-143

[SZ99] A.-S. Sznitman and M.P.W. Zerner (1999). A law of large numbers for random walks in random environment. Ann. Probab. 27, No. 4, 1851-1869

[VZ93] V.A. VAtutin and A.M. Zubkov (1993). Branching processes. II. Probability theory and mathematical statistics, 1. J. Soviet Math. 67, No. 6, 3407-3485

[YMY03] G.P. Yanev, K.V. Mitov and N.M. Yanev (2003). Critical branching regenerative processes with migration. J. Appl. Statist. Sci. 12, $41-54$

[YY95] G.P. YANEV and N.M. YANEV (1995). Critical branching processes with random migration. Lecture Notes in Statist., 99, 36-46, Springer, New York

[Zei04] O. Zeitouni (2004) Random walks in random environment. XXXI Summer School in Probability, St. Flour, 2001, Lecture Notes in Mathematics 1837, pp. 193 - 312, Springer.

[Zer05] M.P.W. Zerner (2005). Multi-excited random walks on integers. Probab. Theory Related Fields 133, $98-122$

[Zer06] M.P.W. ZERNER (2006). Recurrence and transience of excited random walks on $\mathbb{Z}^{d}$ and strips. Elect. Comm. Probab. 11, no. 12, $118-128$ 